\title{
STABILITY OF FLAT INTERFACES DURING SEMIDISCRETE SOLIDIFICATION
}

\begin{abstract}
AndREAS VEESER ${ }^{1}$
Abstract. The stability of flat interfaces with respect to a spatial semidiscretization of a solidification model is analyzed. The considered model is the quasi-static approximation of the Stefan problem with dynamical Gibbs-Thomson law. The stability analysis bases on an argument developed by Mullins and Sekerka for the undiscretized case. The obtained stability properties differ from those with respect to the quasi-static model for certain parameter values and relatively coarse meshes. Moreover, consequences on discretization issues are discussed.
\end{abstract}

Mathematics Subject Classification. 65M12, 65M60.

Received: August 27, 2001. Revised: March 4, 2002.

\section{INTRODUCTION AND MOTIVATION}

The quasi-static approximation of the Stefan problem with dynamical Gibbs-Thomson law is considered. This is a free boundary problem, which models solidification from a pure, possibly supercooled liquid. Flat interfaces moving with appropriate constant velocity constitute "stationary" solutions. These solutions are unstable under certain conditions and are then examples of so-called morphological or Mullins-Sekerka instabilities; see e.g. the books Alexiades and Solomon [1] (Sect. 2.4.G), Gurtin [10] (Sect. 18.2), the review articles Langer [11], Sekerka [15], and the references therein. The purpose of this article is to investigate the stability of flat interfaces for certain spatial discretizations of the afore-mentioned problem. Moreover, consequences of the obtained results on the choice of the discrete spaces and the solver, on a priori error estimates, and on the interpretation of practical simulations are discussed.

In order to motivate such investigation, we recall the isotropic modified Stefan problem in $2 \mathrm{~d}$ and some recent results concerning its numerical solution.

Let $\Omega \subset \mathbb{R}^{2}$ be a bounded domain occupied by a pure substance that may be in solid (superscript $s$ ) or liquid (superscript $l$ ) phase. For any time $t$, the domain $\Omega$ is decomposed in a solid part $\Omega^{s}(t)$, a liquid part $\Omega^{l}(t)$, and a separating interface $\Gamma(t)$. We associate the normal $n$, the normal velocity $V$, and the curvature $\kappa$ of the interface $\Gamma$ with this decomposition. The normal $n$ is pointing into the liquid and the curvature $\kappa$ is positive, if

Keywords and phrases. (Mullins-Sekerka) stability analysis, morphological instabilities, spatial semidiscretization, moving finite elements, phase transitions, surface tension, Stefan condition, dendritic growth, secondary sidebranching.

1 Dipartimento di Matematica, Università degli Studi di Milano, Via C. Saldini 50, 20133 Milano, Italy.

e-mail: veeser@mat.unimi.it

(On leave from) Institut für Angewandte Mathematik, Albert-Ludwigs-Universität Freiburg, Hermann-Herder-Str. 10, 79104 Freiburg, Germany. e-mail: andy@mathematik.uni-freiburg.de 
the solid is convex. The jump across the interface $\Gamma$ with sign convention ' $l$ ' - ' $s$ ' is denoted by $\llbracket \cdot \rrbracket_{s}^{l}$. Moreover, the strictly positive constants $k$ and $c$ are the thermal conductivity and the specific heat (for simplicity, we suppose that these quantities coincide for solid and liquid). Finally, $\beta>0$ is the kinetic coefficient and $\gamma>0$ the solid-liquid surface tension. The (scaled) temperature field $\theta$ and the phase distribution $\left(\Omega^{s}, \Gamma, \Omega^{l}\right)$ of the domain are governed by the equations

$$
\begin{aligned}
c \theta_{t}-\operatorname{div}(k \nabla \theta) & =0 & & \text { in } \Omega^{s}(t) \text { and } \Omega^{l}(t), \\
V+k \llbracket \partial_{n} \theta \rrbracket_{s}^{l} & =0 & & \text { on } \Gamma(t), \\
\beta V+\gamma \kappa+\theta & =0 & & \text { on } \Gamma(t),
\end{aligned}
$$

which are subject to suitable boundary and initial conditions; see e.g. Gurtin [10] (Sect. 17.3.2) and Visintin [19] (Sect. IV.2). Note the following implicit assumption in the dynamical Gibbs-Thomson law (1.1c): the temperature field $\theta$ has no jump across the free boundary $\Gamma$.

Schmidt [14], see also Bänsch and Schmidt [3], proposed and tested a method for the numerical solution of problem (1.1) with adaptive finite elements. Allowing that both the kinetic coefficient $\beta$ and the surface tension $\gamma$ depend smoothly on the normal $n$, simulations of dendritic growth were performed in $2 \mathrm{~d}$ and $3 \mathrm{~d}$. Among others things, it was observed that on coarse meshes secondary side branching takes places, while it does not take place (or may appear at a later time) on finer meshes [14] (Fig. 4.5). Related effects were observed by Fried [9]. In contrast to the parametric approach used in [3,14], Fried relies on a finite element level-set method for a anisotropic variant of the forced mean curvature flow (1.1c).

The fact that the secondary side branching disappears with refining suggests that the observed branching is a numerical artefact. However, if this is true, there is missing an explanation of the secondary side branching on coarse meshes. Such an explanation may be provided by stability analyses of the continuous and (semi)discrete problems.

In [18] the author investigated a semidiscrete finite element method that has the same structure as the approach of $[3,14]$. Error estimates for approximations to the temperature field $\theta$, the phase distribution $\left(\Omega^{s}, \Gamma, \Omega^{l}\right)$, the normal $n$, the normal velocity $V$, and the curvature $\kappa$ are established in $2 \mathrm{~d}$. They cover the case in which both the kinetic coefficient $\beta$ and the surface tension $\gamma$ depend smoothly on the normal $n$. For example, it holds

$$
\sup _{] 0, T[}\left\|\theta_{h}-\theta\right\|_{L_{2}(\Omega)} \leq C h
$$

where $h \leq h^{*}$ denotes the uniform meshsize, $\theta_{h}$ is the discrete temperature field, and $C$ is a constant not depending on $h$. The proof does not allow a realistic estimation of $C$ 's size. However, it sheds some light on the geometric properties of the exact solution that determine $C$ 's size [18] (Rem. 3.1).

It is reasonable to suppose the following: a necessary condition for a moderate size of $C$ is that the stability properties of problem (1.1) and its (semi)discretization are "similar". In this sense, stability analyses of both problems provide a further contribution to a qualitative understanding of $C$ 's size. Furthermore, they may indicate whether or not $C$ has to depend on $T$.

In what follows, we consider the quasi-static approximation of problem (1.1), i.e. the case with $c=0$. This approximation seems to be appropriate if the interface moves slowly with respect to the time-scale of (1.1a). In Section 2 we investigate the stability of flat interfaces by adapting an argument [12] by Mullins and Sekerka to the case of a bounded domain $\Omega$ and kinetic undercooling in the Gibbs-Thomson law (1.1c). The spatial discretization is introduced in Section 3. The interface $\Gamma$ is represented as a graph of a finite element function and the temperature field $\theta$ is approximated by time-depending isoparametric finite elements. In Section 4 we perform the semidiscrete counterpart of Section 2. We compare the results obtained in the continuous and the semidiscrete case and discuss consequences in Section 5. Finally, we show in Section 6 that the results in Section 4 correspond to a (linearized) stability analysis in the classical sense. 


\section{Flat interfaces in the Continuous Case}

We start by presenting the continuous problem which will be considered in the sequel (hereafter, "continuous" often means "non-discrete"). Then, we introduce some stationary solutions and investigate their stability by following an argument due to Mullins and Sekerka [12].

Let $\Omega:=\mathcal{I} \times \mathcal{J}$ with $\mathcal{I}:=] 0,2 \pi[$ and $\mathcal{J}:=]-1,1[$ and denote the outer normal of $\Omega$ by $\nu$. We shall write $z=(x, y) \in \Omega$ and use the letter $D$ to denote the (partial) derivative with respect to $x$ or $y$. Moreover, let $\hat{V}$, $\theta^{s}, \theta^{l} \in \mathbb{R}$ and $T, k, \beta, \gamma>0$ as well as $u_{\text {init }}: \mathcal{I} \rightarrow \mathbb{R}$ be given.

Problem 2.1 (Continuous problem). Find

$$
u: \mathcal{I} \times[0, T[\rightarrow \mathbb{R} \quad \text { and } \quad \theta: \Omega \times[0, T[\rightarrow \mathbb{R}
$$

such that there holds, for all $t \in] 0, T[$,

$$
\begin{aligned}
-\Delta \theta & =0 & & \text { in } \Omega^{s}(t) \text { and } \Omega^{l}(t), \\
V+\hat{V}+k \llbracket \partial_{n} \theta \rrbracket_{s}^{l} & =0 & & \text { on } \Gamma(t), \\
\beta(V+\hat{V})+\gamma \kappa+\theta & =0 & & \text { on } \Gamma(t),
\end{aligned}
$$

as well as

$$
\begin{aligned}
& \theta(x,-1, t)=\theta^{s}, \quad \theta(x, 1, t)=\theta^{l} \quad \text { for all } x \in \mathcal{I}, \\
& \partial_{\nu} \theta(0, y, t)=0, \quad \partial_{\nu} \theta(2 \pi, y, t)=0 \quad \text { for all } y \in \mathcal{J} \text {, } \\
& D u(0, t)=0, \quad D u(2 \pi, t)=0, \\
& |u(x, t)|<1 \quad \text { for all } x \in \mathcal{I},
\end{aligned}
$$

and, for all $x \in \mathcal{I}$,

$$
u(x, 0)=u_{\text {init }}(x) .
$$

Hereafter,

$$
\begin{gathered}
\Omega^{s}(t):=\{z=(x, y) \in \Omega \mid y<u(x, t)\}, \\
\Omega^{l}(t):=\{z=(x, y) \in \Omega \mid y>u(x, t)\}, \\
\Gamma(t):=\operatorname{graph} u(\cdot, t):=\{z=(x, y) \in \Omega \mid y=u(x, t)\}
\end{gathered}
$$

for all $t \in[0, T[$, and

$$
\begin{gathered}
n(z, t):=\frac{(-D u(x, t), 1)}{\sqrt{1+|D u(x, t)|^{2}}}, \quad V(z, t):=\frac{\partial_{t} u(x, t)}{\sqrt{1+|D u(x, t)|^{2}}}, \\
\kappa(z, t):=\frac{-D^{2} u(x, t)}{\left(1+|D u(x, t)|^{2}\right)^{3 / 2}}
\end{gathered}
$$

for all $z=(x, y) \in \Gamma(t), t \in] 0, T[$.

Equations $(2.1 \mathrm{a}-2.1 \mathrm{c})$ restate equations (1.1) with $c=0$ in a frame that moves in vertical direction with constant speed $\hat{V}$. The conditions for the temperature on the (originally moving) boundary are given as follows: on the horizontal walls the temperature is determined by two heaters at temperatures $\theta^{s}$ and $\theta^{l}$, while the vertical walls are isolated. The free boundary $\Gamma(t)$, which is supposed to be a graph over $\mathcal{I}$, has normal contact 
with the vertical walls, but does not touch the horizontal walls. The initial interface $\Gamma(0)$ is given as the graph of the function $u_{\text {init }}$.

Let us mention the following results on local existence and uniqueness of classical solutions of related $(\beta=0$, $\hat{V}=0)$ problems: Escher and Simonett [6,7], Chen et al. [4], and the references therein.

The flat interface

$$
u_{0}(x, t)=0
$$

for all $x \in \mathcal{I}, t \in[0, T[$ and the temperature field

$$
\theta_{0}(z, t)=-\beta \hat{V}+ \begin{cases}p^{s} y, & y \leq 0 \\ p^{l} y, & y \geq 0\end{cases}
$$

for all $z=(x, y) \in \Omega, t \in[0, T[$ with

$$
p^{s}:=-\left(\theta^{s}+\beta \hat{V}\right) \quad \text { and } \quad p^{l}:=\theta^{l}+\beta \hat{V}
$$

constitute a stationary solution of Problem 2.1, iff

$$
\hat{V}=-k\left(p^{l}-p^{s}\right),
$$

that is

$$
(1+2 \beta k) \hat{V}=-k\left(\theta^{s}+\theta^{l}\right) .
$$

Next, supposing that condition (2.5) holds, we investigate the stability of the flat interfaces (2.2a) and their corresponding temperature fields $(2.2 \mathrm{~b})$ in a linearized way.

To this end, set $v_{m}(x):=\cos \left(\frac{m}{2} x\right), x \in \mathcal{I}$, for all $m \in \mathbb{N}_{0}$. The sequence $\left(v_{m}\right)_{m}$ satisfies

$$
-D^{2} v_{m}=\frac{m^{2}}{4} v_{m} \text { in } \mathcal{I}, \quad D v_{m}(0)=0=D v_{m}(2 \pi)
$$

and is a complete orthogonal system for the Hilbert space $L_{2}(\mathcal{I})$. In particular,

$$
L_{2}(\mathcal{I})=\overline{\operatorname{span}\left\{v_{m} \mid m \in \mathbb{N}_{0}\right\}}{ }^{L}(\mathcal{I}) .
$$

Fix $\lambda \in \Lambda:=\left\{m^{2} / 4 \mid m \in \mathbb{N}_{0}\right\}$ and let $v_{\lambda}$ be a corresponding mode or eigenfunction, e.g. the corresponding element from the sequence $\left(v_{m}\right)_{m}$. We consider Problem 2.1 with the perturbed initial value

$$
u_{\text {init }}=\delta v_{\lambda}
$$

where $|\delta|$ is small. We will construct a corresponding approximate solution. To this end, we try the separation ansatz

$$
u_{\delta}(x, t)=\epsilon(t) v_{\lambda}(x)
$$

for all $x \in \mathcal{I}$ and $t \in[0, T$, where the function $\epsilon:[0, T[\rightarrow \mathbb{R}$ has to be determined. In order to make also an ansatz for the temperature field, we introduce the auxiliary functions $w_{\lambda}^{s}, w_{\lambda}^{l}: \mathcal{J} \rightarrow \mathbb{R}$ by

$$
w_{\lambda}^{s}(y):= \begin{cases}y+1, & \lambda=0, \\ \frac{\mathrm{e}^{\sqrt{\lambda} y}}{1-\mathrm{e}^{-2 \sqrt{\lambda}}}-\frac{\mathrm{e}^{-\sqrt{\lambda} y}}{\mathrm{e}^{2 \sqrt{\lambda}}-1}, & \lambda>0,\end{cases}
$$


and

$$
w_{\lambda}^{l}(y):= \begin{cases}-y+1, & \lambda=0 \\ \frac{\mathrm{e}^{-\sqrt{\lambda} y}}{1-\mathrm{e}^{-2 \sqrt{\lambda}}}-\frac{\mathrm{e}^{\sqrt{\lambda} y}}{\mathrm{e}^{2 \sqrt{\lambda}}-1}, & \lambda>0 .\end{cases}
$$

They satisfy

$$
\begin{array}{llrl}
-D^{2} w_{\lambda}^{s}=-\lambda w_{\lambda}^{s} \text { in } \mathcal{J}, & w_{\lambda}^{s}(0)=1, & w_{\lambda}^{s}(-1)=0, \\
-D^{2} w_{\lambda}^{l}=-\lambda w_{\lambda}^{l} \text { in } \mathcal{J}, & w_{\lambda}^{l}(0)=1, & w_{\lambda}^{l}(1)=0 .
\end{array}
$$

Writing

$$
\theta_{\delta}(z, t)=-\beta \hat{V}+ \begin{cases}p^{s} y+A^{s} \epsilon(t) v_{\lambda}(x) w_{\lambda}^{s}(y), & y<u_{\delta}(x, t) \\ p^{l} y+A^{l} \epsilon(t) v_{\lambda}(x) w_{\lambda}^{l}(y), & y>u_{\delta}(x, t)\end{cases}
$$

for all $z=(x, y) \in \Omega$ and $t \in\left[0, T\right.$, we have to determine the real valued constants $A^{s}$ and $A^{l}$ in addition to $\epsilon$; recall the definition of $p^{s}$ and $p^{l}$ in $(2.3)$. Note that $\theta_{\delta}$ is not necessarily continuous.

One immediately verifies that $\left(u_{\delta}, \theta_{\delta}\right)$ satisfies the bulk equations $(2.1 \mathrm{a})$ and the boundary conditions $(2.1 \mathrm{e}$, $2.1 \mathrm{f})$; condition $(2.1 \mathrm{~d})$ is a consequence of $(2.1 \mathrm{~g})$ and the latter is true if $|\epsilon|$ is sufficiently small on $[0, T]$ which will in turn be fulfilled for small $|\delta|$. Therefore, the unknowns $A^{s}, A^{l}$, and $\epsilon$ have to be determined from the Stefan condition (2.1b), the Gibbs-Thomson law (2.1c), the continuity of $\theta_{\delta}$ across $\Gamma_{\delta}(t)=\operatorname{graph} u_{\delta}(\cdot, t)$, and the initial condition $(2.1 \mathrm{~h})$. However, in general, the first three conditions cannot be fulfilled by the ansatz (2.8) exactly. We thus replace these conditions by corresponding approximate ones. Doing so, we use the Landau symbol $O(\cdot)$ to indicate a term that is bounded by a constant times the given argument; the constant may depend on data, $A^{s}, A^{l}$, and $\lambda$ (also through $w_{\lambda}^{s}, w_{\lambda}^{l}$, and $v_{\lambda}$ ), but not on $\delta$. Straight-forward calculations yield the following; see also Alexiades and Solomon [1] (Sect. 2.4.G) or Gurtin [10] (Sect. 18.2).

Provided (2.5), the Stefan condition (2.1b) holds up to terms which are of order $O\left(\epsilon^{2}\right)+O(|\dot{\epsilon} \epsilon|)$, iff

$$
\dot{\epsilon}(t)=k W_{\lambda}\left[A^{s}+A^{l}\right] \epsilon(t)
$$

for all $t \in\left[0, T\left[\right.\right.$. Hereafter, $W_{\lambda}:=D w_{\lambda}^{s}(0)=-D w_{\lambda}^{l}(0)>0$. For later purposes, note that integrating by parts and the properties of $w_{\lambda}^{s}$ yield

$$
W_{\lambda}=\int_{-1}^{0}\left|D w_{\lambda}^{s}\right|^{2}+\lambda\left|w_{\lambda}^{s}\right|^{2} \leq \int_{-1}^{0}|D w|^{2}+\lambda|w|^{2}
$$

for all $w \in H^{1}(]-1,0[)$ with $w(-1)=0$ and $w(0)=1$; an analogue statement holds for the other auxiliary function $w_{\lambda}^{l}$.

The Gibbs-Thomson law (2.1c), where the temperature $\theta_{\delta}$ is evaluated from the solid, holds up to $O\left(\epsilon^{2}\right)+$ $O(|\dot{\epsilon} \epsilon|)$-terms, iff

$$
\beta \dot{\epsilon}(t)=-\left[\gamma \lambda+p^{s}+A^{s}\right] \epsilon(t)
$$

for all $t \in\left[0, T\right.$. Similarly, the Gibbs-Thomson law (2.1c), where the temperature $\theta_{\delta}$ is evaluated from the liquid, holds up to $O\left(\epsilon^{2}\right)+O(|\dot{\epsilon} \epsilon|)$, iff

$$
\beta \dot{\epsilon}(t)=-\left[\gamma \lambda+p^{l}+A^{l}\right] \epsilon(t)
$$

for all $t \in[0, T[$. 
The ordinary differential equations (2.9) and (2.11) can be satisfied instantaneously only if

$$
\beta k W_{\lambda}\left[A^{s}+A^{l}\right]=-\left[\gamma \lambda+p^{s}+A^{s}\right]=-\left[\gamma \lambda+p^{l}+A^{l}\right] .
$$

This is fulfilled for (recall (2.4) and $W_{\lambda}>0$ )

$$
\begin{aligned}
A^{s} & =-\frac{\gamma \lambda+p^{s}+\beta W_{\lambda} \hat{V}}{1+2 \beta k W_{\lambda}}, \\
A^{l} & =-\frac{\gamma \lambda+p^{l}-\beta W_{\lambda} \hat{V}}{1+2 \beta k W_{\lambda}}
\end{aligned}
$$

which leads to

$$
\epsilon(t)=\delta \mathrm{e}^{\mu t}
$$

with

$$
\mu=\frac{k W_{\lambda}}{1+2 \beta k W_{\lambda}}\left(-2 \gamma \lambda-p^{l}-p^{s}\right)
$$

for all $t \in\left[0, T\right.$. Finally, note that we have $|\epsilon| \leq|\delta| \mathrm{e}^{\mu T}$ on $[0, T]$.

We summarize:

Theorem 2.1 (Approximate continuous solutions). Fix an eigenvalue $\lambda \in \Lambda$ of $-D^{2}$ with natural boundary conditions, let $v_{\lambda}$ be a corresponding mode, and suppose that (2.5) holds.

Then ansatz (2.8) with (2.12) provides a family $\left(\theta_{\delta}, u_{\delta}\right)_{\delta}$ such that

- if $|\delta|$ is sufficiently small, the functions $\theta_{\delta}$ and $u_{\delta}$ satisfy (2.1a, 2.1d-2.1h) with initial value $u_{\mathrm{init}}=\delta v_{\lambda}$,

- for $\delta \rightarrow 0$, the functions $\theta_{\delta}$ and $u_{\delta}$ are continuous apart from an $O\left(\delta^{2}\right)$-jump of $\theta_{\delta}$ across the free boundary and they satisfy, for all $t \in[0, T[$,

$$
\begin{aligned}
\left.V_{\delta}+\hat{V}+k \llbracket \frac{\partial \theta_{\delta}}{\partial n_{\delta}}\right]_{s}^{l} & =O\left(\delta^{2}\right) & & \text { on } \Gamma_{\delta}(t), \\
\beta\left(V_{\delta}+\hat{V}\right)+\gamma \kappa_{\delta}+\theta_{\delta} & =O\left(\delta^{2}\right) & & \text { on } \Gamma_{\delta}(t),
\end{aligned}
$$

where $\theta_{\delta}$ in the last identity can be evaluated from the solid or the liquid and $V_{\delta}, n_{\delta}, \Gamma_{\delta}$, and $\kappa_{\delta}$ are defined in an analogue way to $V, n, \Gamma$, and $\kappa$. Here $O\left(\delta^{2}\right)$ indicates terms that are bounded by $\delta^{2}$ times a constant depending on data, $\lambda$, and the choice of $v_{\lambda}$.

We may read Theorem 2.1 in the following way. The initial value $u_{\text {init }}=0$ is perturbed in direction of the mode $v_{\lambda}$ with order $\delta$. But instead of determining the exact solution with respect to the perturbed initial value, we give an approximate solution which solves Problem 2.1 up to terms of order $\delta^{2}$. Since the order of these terms is higher than the perturbation of the initial value, one may hope that, for $\delta \rightarrow 0$, the approximate solution captures the stability of the considered perturbation; compare also with Section 6 . This interpretation, together with (2.6), suggests that the amplification rate $\mu$ in (2.12d) sheds some light on the stability properties of the stationary solution (2.2).

We postpone a more detailed discussion of formula (2.12d) to Section 5 . Here, we just recall that an investigation of $\mu$ 's sign indicates: undercooling of the liquid $\left(p^{l}<0\right)$ and superheating of the solid $\left(p^{s}<0\right)$ are destabilizing, while surface tension $\gamma>0$ stabilizes for modes corresponding to big eigenvalues $\lambda$. 


\section{Semidiscretization}

In this section we introduce a spatial discretization of Problem 2.1 with the help of finite elements. The discrete counterpart of the function $u$ is continuous and piecewise polynomial, while the discrete temperature field is constructed by means of moving (or time-depending isoparametric) elements with an underlying tensor product structure.

We first derive a weak formulation of Problem 2.1, which is suitable for finite elements; compare with [3, $5,14,18]$. Let $\chi: \Omega \rightarrow \mathbb{R}$ be a test function with $\chi_{\mid \mathcal{I} \times \partial \mathcal{J}=0}$. Multiplying the bulk equations $(2.1 \mathrm{a})$ with $k \chi$, integrating over $\Omega^{s}(t)$ and $\Omega^{l}(t)$, integrating by parts, using the boundary conditions $(2.1 \mathrm{e})$ and the Stefan condition $(2.1 \mathrm{~b})$ yields

$$
\int_{\Omega} k \nabla \theta \cdot \nabla \chi=\int_{\mathcal{I}}\left(\frac{\partial_{t} u}{\sqrt{1+|D u|^{2}}}+\hat{V}\right) \chi(u) \sqrt{1+|D u|^{2}} .
$$

Hereafter, $\chi(u)$ is defined by $\chi(u)(x):=\chi(x, u(x)), x \in \mathcal{I}$.

Similarly, let $\varphi: \mathcal{I} \rightarrow \mathbb{R}$ be a test function. Multiplying the Gibbs-Thomson law (2.1c) with $\varphi$, integrating over $\mathcal{I}$, using $\kappa=-D\left(D u / \sqrt{1+|D u|^{2}}\right)$, integrating by parts the principal term, and using the boundary conditions (2.1f) gives

$$
\beta \int_{\mathcal{I}} \frac{\partial_{t} u}{\sqrt{1+|D u|^{2}}} \varphi+\gamma \int_{\mathcal{I}} \frac{D u}{\sqrt{1+|D u|^{2}}} D \varphi=-\int_{\mathcal{I}}[\theta(u)+\beta \hat{V}] \varphi
$$

It remains to choose discrete spaces in the weak equations (3.1) and (3.2); as usual, we shall use the subscript $h$ to indicate discrete quantities.

We first introduce those for the weak Gibbs-Thomson law (3.2). Let $q \in \mathbb{N}$ and $X_{h}$ be the space of Lagrange elements of order $q$ over some fixed partition of $\mathcal{I}$, i.e. the space of continuous functions that are piecewise polynomial of degree less than or equal to $q$. We may number the nodes of $X_{h}$ in the following way:

$$
0=x_{0}<x_{1}<\cdots<x_{I-1}<x_{I}=2 \pi .
$$

The nodal basis for $X_{h}$ is given by the functions $\varphi_{i} \in X_{h}, i=0, \ldots, I$, with

$$
\varphi_{i}\left(x_{m}\right)=\delta_{i, m}:=\left\{\begin{array}{ll}
1, & \text { if } i=m, \\
0, & \text { otherwise }
\end{array} \text { for } m=0, \ldots, I .\right.
$$

We turn to the discrete spaces for the weak bulk equation (3.1). To this end, we first construct a tensor product space. One factor is the space $X_{h}$, the other one is the space $Y_{h}$ of Lagrange elements of order $r$ over some fixed partition of $\mathcal{J}$ which vanish in $\partial \mathcal{J}$. We suppose that the point $0 \in \mathcal{J}$ is the common boundary of two elements of $Y_{h}$ and number its nodes in the following way:

$$
-1<y_{\underline{J}}<\cdots<y_{0}=0<y_{1}<\cdots<y_{\bar{J}}<1 .
$$

The nodal basis for $Y_{h}$ is denoted by $\left(\psi_{j}\right)_{j=\underline{J}, \ldots, \bar{J}}$. As announced, we define the tensor product space

$$
Z_{h}(0):=X_{h} \otimes Y_{h},
$$

which has the basis $\left(\hat{\chi}_{i j}\right)_{i j}$, where

$$
\hat{\chi}_{i j}(z):=\left(\varphi_{i} \otimes \psi_{j}\right)(x, y)=\varphi_{i}(x) \psi_{j}(y)
$$

for $z=(x, y) \in \Omega, i=0, \ldots, I$, and $j=\underline{J}, \ldots, \bar{J}$. 
Next, we construct a map from $\Omega$ to $\Omega$, which will depend on the discrete free boundary; with the help of this map and the space $Z_{h}(0)$ we shall define the discrete test space for the weak bulk equation (3.1). To this end, let $y_{*}:=y_{-r}$ and $y^{*}:=y_{r}$ denote the endpoints of the union of the two elements containing the point $y_{0}=0$. Furthermore, we set

$$
\omega_{\max }:=\frac{1}{2} \min \left\{-y_{*}, y^{*}\right\}>0
$$

and define the function

$$
g(\cdot ; \omega): \mathcal{J} \rightarrow \mathcal{J}, \quad y \mapsto \begin{cases}\omega+\frac{y_{*}-\omega}{y_{*}} y, & \text { if } y \in\left[y_{*}, 0\right], \\ \omega+\frac{y^{*}-\omega}{y^{*}} y, & \text { if } y \in\left[0, y^{*}\right] \\ y, & \text { elsewhere, }\end{cases}
$$

which depends on the parameter $\omega \in\left[-\omega_{\max }, \omega_{\max }\right]$. Moreover, we introduce the following subset

$$
B_{h}:=\left\{\varphi_{h} \in X_{h}\left|\left\|\varphi_{h}\right\|_{0, \infty ; \mathcal{I}}:=\sup _{\mathcal{I}}\right| \varphi_{h} \mid<\omega_{\max }\right\}
$$

of $X_{h}$ and define

$$
G\left(\cdot ; w_{h}\right): \Omega \rightarrow \Omega, \quad z=(x, y) \mapsto\left(x, g\left(y ; w_{h}(x)\right)\right)
$$

for $w_{h} \in B_{h}$. Both maps $g(\cdot ; \omega)$ and $G\left(\cdot ; w_{h}\right)$ are bijections for the allowed parameters values; their inverses are denoted by $g^{-1}(\cdot ; \omega)$ and $G^{-1}\left(\cdot ; w_{h}\right)$, respectively. All four maps are Lipschitz continuous. Furthermore, $G\left(\cdot ; w_{h}\right), w_{h} \in B_{h}$, satisfies

$$
\begin{gathered}
G\left(\mathcal{I} \times\left[-1, y_{*}\right] ; w_{h}\right)=\mathcal{I} \times\left[-1, y_{*}\right], \\
G\left(\mathcal{I} \times\left[y_{*}, y^{*}\right] ; w_{h}\right)=\mathcal{I} \times\left[y_{*}, y^{*}\right], \quad G\left(\mathcal{I} \times\{0\} ; w_{h}\right)=\operatorname{graph} w_{h} \\
G\left(\mathcal{I} \times\left[y^{*}, 1\right] ; w_{h}\right)=\mathcal{I} \times\left[y^{*}, 1\right] .
\end{gathered}
$$

Finally, for $w_{h} \in B_{h}$, we define the discrete space

$$
Z_{h}\left(w_{h}\right):=\left\{\hat{\chi}_{h} \circ G\left(w_{h}\right)^{-1} \mid \hat{\chi}_{h} \in Z_{h}(0)\right\}
$$

by using the abbreviation $G\left(w_{h}\right)^{-1}:=G^{-1}\left(\cdot ; w_{h}\right)$. Note that, thanks to $G(\cdot ; 0)=\mathrm{id}_{\Omega}$, the definition $(3.9)$ is consistent with the one in (3.3). A basis for $Z_{h}\left(w_{h}\right)$ is given by $\hat{\chi}_{i j} \circ G\left(w_{h}\right)^{-1}, i=0, \ldots, I, j=\underline{J}, \ldots, \bar{J}$.

Let the discrete initial interface be given as graph of a function $u_{h \text {,init }} \in B_{h}$ and define

$$
\theta_{b}(z):=\theta^{l} \frac{1+y}{2}+\theta^{s} \frac{1-y}{2}
$$

for all $z=(x, y) \in \Omega$. In the next section we will investigate stability properties of the following semidiscrete version of Problem 2.1.

Problem 3.1 (Semidiscrete problem). Find

$$
u_{h}:\left[0, T\left[\rightarrow X_{h} \quad \text { and } \quad \theta_{h}:\left[0, T\left[\rightarrow \theta_{b}+\left\{\chi \in H^{1}(\Omega) \mid \chi_{\mid \mathcal{I} \times \partial \mathcal{J}}=0\right\}\right.\right.\right.\right.
$$


such that there holds, for all $t \in] 0, T[$,

$$
\begin{gathered}
u_{h}(t) \in B_{h}, \\
\theta(t) \in \theta_{b}+Z_{h}\left(u_{h}(t)\right),
\end{gathered}
$$

and

$$
\begin{gathered}
\int_{\Omega} k \nabla \theta_{h} \cdot \nabla \chi_{h}=\int_{\mathcal{I}}\left(\frac{\partial_{t} u_{h}}{\sqrt{1+\left|D u_{h}\right|^{2}}}+\hat{V}\right) \chi_{h}\left(u_{h}\right) \sqrt{1+\left|D u_{h}\right|^{2}} \\
\beta \int_{\mathcal{I}} \frac{\partial_{t} u_{h}}{\sqrt{1+\left|D u_{h}\right|^{2}}} \varphi_{h}+\gamma \int_{\mathcal{I}} \frac{D u_{h}}{\sqrt{1+\left|D u_{h}\right|^{2}}} D \varphi_{h}=-\int_{\mathcal{I}}\left[\theta_{h}\left(u_{h}\right)+\beta \hat{V}\right] \varphi_{h}
\end{gathered}
$$

for all $\chi_{h} \in Z_{h}\left(u_{h}(t)\right)$ and $\varphi_{h} \in X_{h}$, as well as

$$
u_{h}(0)=u_{h, \text { init }}
$$

Later (see Theorem 6.1), we will show that, for sufficiently small $T>0$ and $u_{h \text {,init }} \in B_{h}$, the semidiscrete Problem 3.1 admits a unique solution.

We conclude this section with a remark on the use of moving (or time-depending isoparametric) elements for the temperature field. On the one hand, this has the disadvantage that the semidiscrete solution concept may breakdown (even for a more sophisticated use of moving elements) although there is no break down in the continuous solution. But, on the other hand, there is the following advantage with regard to our purposes. The discrete space $Z_{h}\left(u_{h}(t)\right)$ allows for a jump in the discrete heat flow across the moving support of the right-hand side in the weak bulk equation (3.10c). Discretizations with time-independent elements as in [3,14,18] does not exhibit this property. However, the continuous (i.e. non-discrete) heat flow jumps across the moving support of the right-hand side in (3.1). In view of this observation, one may expect that the semidiscrete Problem 3.1 with moving elements has a better chance to mimic the stability properties of the continuous Problem 2.1 than other (semi)discretizations with time-independent elements.

\section{Flat interfaces in the SEMidiscrete CASE}

The flat interfaces (2.2a) and their corresponding temperature fields (2.2b) with (2.5) are solutions of the semidiscrete Problem 3.1, too. This offers the possibility to investigate the stability of these stationary solutions in the context of the semidiscrete Problem 3.1 and then to compare the results with those in the continuous case. The first step is carried out in this section, while the second one will be done in the next section.

We take the approach of Section 2 as a guideline. Let us consider the operator $-D_{h}^{2}: X_{h} \rightarrow X_{h}$ which is defined by means of the Riesz representation theorem as follows: for $v_{h} \in X_{h}$, let $-D_{h}^{2} v_{h}$ be the unique element in $X_{h}$ with

$$
\int_{\mathcal{I}}\left(-D_{h}^{2} v_{h}\right) \varphi_{h}=\int_{\mathcal{I}} D v_{h} D \varphi_{h}
$$

for all $\varphi_{h} \in X_{h}$. The operator $-D_{h}^{2}$ is symmetric (with respect to the $L_{2}(\mathcal{I})$-scalar product) and positive semidefinite. Consequently, its spectrum $\Lambda_{h}$ is real and nonnegative and appropriately chosen eigenfunctions constitute an orthonormal basis of $X_{h} \subset L_{2}(\mathcal{I})$. Let $v_{h, \lambda}$ be such an eigenfunction and denote its corresponding eigenvalue by $\lambda$. 
Similar to Section 2, we consider Problem 3.1 with the perturbed initial value

$$
u_{h, \text { init }}=\delta v_{h, \lambda},
$$

where $|\delta|$ is small. The counterpart of $(2.8 \mathrm{a})$ is the ansatz

$$
u_{h, \delta}(x, t)=\epsilon_{h}(t) v_{h, \lambda}(x)
$$

for all $x \in \mathcal{I}$ and $t \in\left[0, T\left[\right.\right.$, where the function $\epsilon_{h}:[0, T[\rightarrow \mathbb{R}$ will be determined later. For the ansatz of the semidiscrete temperature, we introduce the discrete counterparts of $w_{\lambda}^{s}$ and $w_{\lambda}^{l}$. Setting

$$
Y_{h}^{s}:=\left\{\psi_{h \mid[-1,0]} \mid \psi_{h} \in Y_{h}\right\} \quad \text { and } \quad Y_{h}^{l}:=\left\{\psi_{h \mid[0,1]} \mid \psi_{h} \in Y_{h}\right\}
$$

we define $w_{h, \lambda}^{s} \in Y_{h}^{s}$ and $w_{h, \lambda}^{l} \in Y_{h}^{l}$ by

$$
w_{h, \lambda}^{s}(0)=1, \quad \int_{-1}^{0} D w_{h, \lambda}^{s} D \psi_{h}+\lambda w_{h, \lambda}^{s} \psi_{h}=0
$$

for all $\psi_{h} \in Y_{h}^{s}$ with $\psi_{h}(0)=0$ and

$$
w_{h, \lambda}^{l}(0)=1, \quad \int_{0}^{1} D w_{h, \lambda}^{l} D \psi_{h}+\lambda w_{h, \lambda}^{l} \psi_{h}=0
$$

for all $\psi_{h} \in Y_{h}^{l}$ with $\psi_{h}(0)=0$, respectively (since $\lambda \geq 0$, the functions $w_{h, \lambda}^{s}$ and $w_{h, \lambda}^{l}$ exist and are uniquely determined). Note that there holds

$$
\int_{-1}^{0} D w_{h, \lambda}^{s} D \psi_{h}=-\lambda \int_{-1}^{0} w_{h, \lambda}^{s} \psi_{h}+W_{h, \lambda}^{s} \psi_{h}(0)
$$

and

$$
\int_{0}^{1} D w_{h, \lambda}^{l} D \psi_{h}=-\lambda \int_{0}^{1} w_{h, \lambda}^{l} \psi_{h}-W_{h, \lambda}^{l} \psi_{h}(0)
$$

for all $\psi_{h} \in Y_{h}^{s}$ and $\psi_{h} \in Y_{h}^{l}$, respectively, with

$$
W_{h, \lambda}^{s}:=\int_{-1}^{0} D w_{h, \lambda}^{s} D \psi_{0}+\lambda w_{h, \lambda}^{s} \psi_{0} \quad \text { and } \quad W_{h, \lambda}^{l}:=-\int_{0}^{1} D w_{h, \lambda}^{l} D \psi_{0}+\lambda w_{h, \lambda}^{l} \psi_{0}
$$

(recall that $\psi_{0}$ is the basis function corresponding to the node $0 \in \mathcal{J}$ ). Note also that (4.2a), the Cauchy-Schwarz inequality, $w_{h, \lambda}^{s}(0)=1$, and $w_{h, \lambda}^{s}(-1)=0$ imply

$$
W_{h, \lambda}^{s}=\int_{-1}^{0}\left|D w_{h, \lambda}^{s}\right|^{2}+\lambda\left|w_{h, \lambda}^{s}\right|^{2} \geq \int_{-1}^{0} D w_{h, \lambda}^{s}=1
$$

similarly, one obtains

$$
W_{h, \lambda}^{l}=-\int_{0}^{1}\left|D w_{h, \lambda}^{l}\right|^{2}+\lambda\left|w_{h, \lambda}^{l}\right|^{2} \leq-\int_{0}^{1}\left|D w_{h, \lambda}^{l}\right| \leq \int_{0}^{1} D w_{h, \lambda}^{l}=-1 .
$$


Finally, let us observe that, similarly to (2.10),

$$
W_{h, \lambda}^{s}=\int_{-1}^{0}\left|D w_{h, \lambda}^{s}\right|^{2}+\lambda\left|w_{h, \lambda}^{s}\right|^{2} \leq \int_{-1}^{0}\left|D w_{h}\right|^{2}+\lambda\left|w_{h}\right|^{2}
$$

for all $w_{h} \in Y_{h}^{s}$ with $w_{h}(0)=1$ and an analogue statement for $w_{h, \lambda}^{l}$ hold.

Using the functions $w_{h, \lambda}^{s}, w_{h, \lambda}^{l}$ and supposing conditions (2.5) and (3.10a), we write

$$
\theta_{h, \delta}(z, t):=-\beta \hat{V}+ \begin{cases}p^{s} y+A_{h}^{s} \epsilon_{h}(t) v_{h, \lambda}(x) w_{h, \lambda}^{s}\left(g^{-1}\left(y ; u_{h, \delta}(x, t)\right)\right), & \text { if } y<u_{h, \delta}(x, t) \\ p^{l} y+A_{h}^{l} \epsilon_{h}(t) v_{h, \lambda}(x) w_{h, \lambda}^{l}\left(g^{-1}\left(y ; u_{h, \delta}(x, t)\right)\right), & \text { if } y>u_{h, \delta}(x, t)\end{cases}
$$

for all $z=(x, y) \in \Omega$ and $t \in\left[0, T\right.$, where, apart from $\epsilon_{h}$, the constants $A_{h}^{s}$ and $A_{h}^{l}$ are to be determined. Note that $\theta_{h, \delta}$ does not verify condition (3.10b) necessarily. However, there holds

$$
\theta_{h, \delta}(x, \pm 1, t)=\theta_{b}(x, \pm 1)
$$

for all $x \in \mathcal{I}$ thanks to (2.3). In addition,

$$
\begin{aligned}
\left(\theta_{h, \delta}(t)-\theta_{b}\right) \circ G\left(x, y ; u_{h, \delta}(t)\right)=-\beta \hat{V}+p^{s} g\left(y ; u_{h, \delta}(x, t)\right) & +A_{h}^{s} \epsilon_{h}(t) v_{h, \lambda}(x) w_{h, \lambda}^{s}(y) \\
& -\frac{\theta^{l}}{2}\left[1+g\left(y ; u_{h, \delta}(x, t)\right)\right]-\frac{\theta^{s}}{2}\left[1-g\left(y ; u_{h, \delta}(x, t)\right)\right]
\end{aligned}
$$

and

$$
g\left(y ; u_{h, \delta}(x, t)\right)= \begin{cases}y, & \text { if } y \in\left[-1, y_{*}\right] \\ u_{h, \delta}(x, t)+y-\frac{u_{h, \delta}(x, t)}{y_{*}} y, & \text { if } y \in\left[y_{*}, 0\right]\end{cases}
$$

for all $t \in\left[0, T\left[, x \in \mathcal{I}\right.\right.$, and $y \in[-1,0]$ as well as similar formulas for all $y \in[0,1]$. Therefore, since $u_{h, \delta}(t)$, $v_{h, \lambda} \in X_{h}$ and $w_{h, \lambda}^{s} \in Y_{h}^{s}, w_{h, \lambda}^{l} \in Y_{h}^{l}$, the only missing point for $(3.10 \mathrm{~b})$ is the continuity across graph $u_{h, \delta}(t)$, the discrete free boundary. The latter one is given, iff

$$
p^{s}+A_{h}^{s}=p^{l}+A_{h}^{l}
$$

Next, we investigate the discrete bulk equations (3.10c) and the semidiscrete Gibbs-Thomson law (3.10d) as to determine $A_{h}^{s}, A_{h}^{l}$, and $\epsilon_{h}$. In this section we use a variant $O_{V}(\cdot ; \cdot)$ of the Landau symbol to indicate a linear functional in the second argument whose norm in the dual of the normed vector space $V$ is bounded by a constant times the first argument; the constant may depend on data, $A_{h}^{s}, A_{h}^{l}$, $\lambda$, the choice of $v_{h, \lambda}$, and the discrete spaces $X_{h}$ and $Y_{h}$. In this regard it is convenient to endow $Z_{h}(t):=Z_{h}\left(u_{h, \delta}(t)\right), t \in[0, T[$, with the norm

$$
Z_{h}(t) \ni \chi_{h} \mapsto\left\|\chi_{h}\right\|_{1, \infty ; \Omega}:=\sup _{\Omega}\left|\chi_{h}\right|+\sup _{\Omega}\left|\nabla \chi_{h}\right|
$$

and $X_{h}$ with the norm

$$
X_{h} \ni \varphi_{h} \mapsto\left\|\varphi_{h}\right\|_{1,2 ; \mathcal{I}}:=\left(\left\|\varphi_{h}\right\|_{0,2 ; \mathcal{I}}^{2}+\left\|D \varphi_{h}\right\|_{0,2 ; \mathcal{I}}^{2}\right)^{1 / 2}
$$

where $\|\cdot\|_{0,2 ; \mathcal{I}}$ denotes the norm of $L_{2}(\mathcal{I})$. 
In order to derive a counterpart of (2.9), we split the discrete temperature field

$$
\theta_{h, \delta}=\theta_{h, \delta}^{0}+\theta_{h, \delta}^{1}
$$

into the parts

$$
\theta_{h, \delta}^{0}(z, t):=-\beta \hat{V}+ \begin{cases}p^{s} y, & \text { if } y<u_{h, \delta}(x, t) \\ p^{l} y, & \text { if } y>u_{h, \delta}(x, t)\end{cases}
$$

and

$$
\theta_{h, \delta}^{1}(z, t):=\epsilon_{h}(t) v_{h, \lambda}(x) \begin{cases}A_{h}^{s} w_{h, \lambda}^{s}\left(g^{-1}\left(y ; u_{h, \delta}(x, t)\right)\right), & \text { if } y<u_{h, \delta}(x, t), \\ A_{h}^{l} w_{h, \lambda}^{l}\left(g^{-1}\left(y ; u_{h, \delta}(x, t)\right)\right), & \text { if } y>u_{h, \delta}(x, t)\end{cases}
$$

for all $z=(x, y) \in \Omega$ and $t \in\left[0, T\left[\right.\right.$. Let $t \in\left[0, T\left[\right.\right.$ and $\chi_{h} \in Z_{h}(t)$. Integrating by parts, $\chi_{h \mid \mathcal{I} \times \partial \mathcal{J}}=0$, $\partial_{\nu} \theta_{h, \delta \mid \partial \mathcal{I} \times \mathcal{J}}^{0}=0$, and (2.4) yield

$$
\int_{\Omega} k \nabla \theta_{h, \delta}^{0} \cdot \nabla \chi_{h}=\int_{\mathcal{I}} \hat{V} \chi_{h}\left(u_{h, \delta}\right) \sqrt{1+\left|D u_{h, \delta}\right|^{2}}+O_{Z_{h}(t)}\left(\epsilon_{h}^{2} ; \chi_{h}\right)
$$

in $t$. The analogue identity for $\theta_{h, \delta}^{1}$ is more involved. By changing variables, see e.g. [8] (Sect. 3.3, Th. 2), and using the chain rule, e.g. [20] (Sect. 2.2.2), we obtain

$$
\begin{aligned}
\int_{\Omega} k \nabla \theta_{h, \delta}^{1} \cdot \nabla \chi_{h}= & \int_{\Omega} k \nabla\left[\theta_{h, \delta}^{1} \circ G\left(u_{h, \delta}\right)\right] \cdot \nabla\left[\chi_{h} \circ G\left(u_{h, \delta}\right)\right] \\
& +\int_{\Omega} k \nabla\left[\theta_{h, \delta}^{1} \circ G\left(u_{h, \delta}\right)\right] \cdot \nabla\left[\chi_{h} \circ G\left(u_{h, \delta}\right)\right]\left(J G\left(u_{h, \delta}\right)-1\right) \\
& +\int_{\Omega} k \nabla\left[\theta_{h, \delta}^{1} \circ G\left(u_{h, \delta}\right)\right] \cdot\left(\nabla \chi_{h}\right) \circ G\left(u_{h, \delta}\right)\left[E-D G\left(u_{h, \delta}\right)\right] J G\left(u_{h, \delta}\right) \\
& +\int_{\Omega} k\left(\nabla \theta_{h, \delta}^{1}\right) \circ G\left(u_{h, \delta}\right)\left[E-D G\left(u_{h, \delta}\right)\right] \cdot\left(\nabla \chi_{h}\right) \circ G\left(u_{h, \delta}\right) J G\left(u_{h, \delta}\right) \\
= & : \int_{\Omega} k \nabla\left[\theta_{h, \delta}^{1} \circ G\left(u_{h, \delta}\right)\right] \cdot \nabla\left[\chi_{h} \circ G\left(u_{h, \delta}\right)\right]+R_{h, \delta}\left(\chi_{h}\right),
\end{aligned}
$$

where $E$ denotes the matrix of the identity in $\mathbb{R}^{2}$ and $J G\left(u_{h, \delta}\right):=\left|\operatorname{det} D G\left(u_{h, \delta}\right)\right|$. Let us check that the first term on the right-hand side of (4.10) is convenient for our purposes. Note that

$$
\theta_{h, \delta}^{1}\left(G\left(z ; u_{h, \delta}(t)\right), t\right)=\epsilon_{h}(t) v_{h, \lambda}(x) \begin{cases}A_{h}^{s} w_{h, \lambda}^{s}(y), & \text { if } y<0 \\ A_{h}^{l} w_{h, \lambda}^{l}(y), & \text { if } y>0\end{cases}
$$


for all $z=(x, y) \in \Omega$. Assuming for a moment that $\chi_{h} \circ G\left(u_{h, \delta}\right)=\varphi_{h} \otimes \psi_{h}$ with $\varphi_{h} \in X_{h}$ and $\psi_{h} \in Y_{h}$ and using (4.2a), we observe

$$
\begin{aligned}
\int_{\mathcal{I} \times]-1,0[} k \nabla\left[\theta_{h, \delta}^{1} \circ G\left(u_{h, \delta}\right)\right] \cdot \nabla\left[\chi_{h} \circ G\left(u_{h, \delta}\right)\right] \\
=k A_{h}^{s} \epsilon_{h} \int_{\mathcal{I}} \int_{-1}^{0} \partial_{x}\left(v_{h, \lambda} \otimes w_{h, \lambda}^{s}\right) \partial_{x}\left(\varphi_{h} \otimes \psi_{h}\right)+\partial_{y}\left(v_{h, \lambda} \otimes w_{h, \lambda}^{s}\right) \partial_{y}\left(\varphi_{h} \otimes \psi_{h}\right) \\
=k A_{h}^{s} \epsilon_{h}\left(\int_{\mathcal{I}} D v_{h, \lambda} D \varphi_{h} \int_{-1}^{0} w_{h, \lambda}^{s} \psi_{h}+\int_{\mathcal{I}} v_{h, \lambda} \varphi_{h} \int_{-1}^{0} D w_{h, \lambda}^{s} D \psi_{h}\right) \\
=k A_{h}^{s} \epsilon_{h}\left(\lambda \int_{\mathcal{I}} v_{h, \lambda} \varphi_{h} \int_{-1}^{0} w_{h, \lambda}^{s} \psi_{h}-\lambda \int_{\mathcal{I}} v_{h, \lambda} \varphi_{h} \int_{-1}^{0} w_{h, \lambda}^{s} \psi_{h}\right. \\
\left.\quad+W_{h, \lambda}^{s} \psi_{h}(0) \int_{\mathcal{I}} v_{h, \lambda} \varphi_{h}\right) \\
=k W_{h, \lambda}^{s} A_{h}^{s} \epsilon_{h} \int_{\mathcal{I}} v_{h, \lambda} \varphi_{h} \psi_{h}(0)=k W_{h, \lambda}^{s} A_{h}^{s} \epsilon_{h} \int_{\mathcal{I}} v_{h, \lambda} \chi_{h}\left(u_{h, \delta}\right) .
\end{aligned}
$$

Since both sides of this identity depend linearly on $\chi_{h}$, it holds in general. Similarly, we derive

$$
\int_{\mathcal{I} \times] 0,1[} k \nabla\left[\theta_{h, \delta}^{1} \circ G\left(u_{h, \delta}\right)\right] \cdot \nabla\left[\chi_{h} \circ G\left(u_{h, \delta}\right)\right]=-k W_{h, \lambda}^{l} A_{h}^{l} \epsilon_{h} \int_{\mathcal{I}} v_{h, \lambda} \chi_{h}\left(u_{h, \delta}\right)
$$

with the help of $(4.2 \mathrm{~b})$. Summing the last two identities gives

$$
\int_{\Omega} k \nabla\left[\theta_{h, \delta}^{1} \circ G\left(u_{h, \delta}\right)\right] \cdot \nabla\left[\chi_{h} \circ G\left(u_{h, \delta}\right)\right]=k\left(W_{h, \lambda}^{s} A_{h}^{s}-W_{h, \lambda}^{l} A_{h}^{l}\right) \epsilon_{h} \int_{\mathcal{I}} v_{h, \lambda} \chi_{h}\left(u_{h, \delta}\right) .
$$

Therefore, noting that

$$
\int_{\mathcal{I}} \frac{\partial_{t} u_{h, \delta}}{\sqrt{1+\left|D u_{h, \delta}\right|^{2}}} \chi_{h}\left(u_{h, \delta}\right) \sqrt{1+\left|D u_{h, \delta}\right|^{2}}=\dot{\epsilon}_{h} \int_{\mathcal{I}} v_{h, \lambda} \chi_{h}\left(u_{h, \delta}\right)
$$

we obtain that

$$
\int_{\Omega} k \nabla\left[\theta_{h, \delta}^{1} \circ G\left(u_{h, \delta}\right)\right] \cdot \nabla\left[\chi_{h} \circ G\left(u_{h, \delta}\right)\right]=\int_{\mathcal{I}} \frac{\partial_{t} u_{h, \delta}}{\sqrt{1+\left|D u_{h, \delta}\right|^{2}}} \chi_{h}\left(u_{h, \delta}\right) \sqrt{1+\left|D u_{h, \delta}\right|^{2}}
$$

holds, iff

$$
\dot{\epsilon}_{h}=k\left[W_{h, \lambda}^{s} A_{h}^{s}-W_{h, \lambda}^{l} A_{h}^{l}\right] \epsilon_{h} .
$$

So, we need to show that $R_{h, \delta}\left(\chi_{h}\right)$ in $(4.10)$ is $O_{Z_{h}(t)}\left(\epsilon_{h}^{2} ; \chi_{h}\right)$. To this end, we derive further properties of $G$ in the region $\mathcal{I} \times\left[y_{*}, y^{*}\right]$. It holds

$$
D G\left(x, y ; u_{h, \delta}(t)\right)=\left(\begin{array}{cc}
1 & 0 \\
\epsilon_{h}(t)\left(1-\frac{y}{y_{*}}\right) D v_{h, \lambda}(x) & 1-\epsilon_{h}(t) \frac{v_{h, \lambda}(x)}{y_{*}}
\end{array}\right)
$$

for almost all $(x, y) \in \mathcal{I} \times] y_{*}, 0\left[\right.$ and a similar (replace $y_{*}$ by $y^{*}$ ) formula for almost all $\left.(x, y) \in \mathcal{I} \times\right] 0, y^{*}[$. Let us suppose that condition (3.10a) holds. This will be true for small $|\delta|$; see (4.15c) below. Then (3.4) and (3.6) 
imply the following estimates almost everywhere in $\Omega$ :

$$
\begin{aligned}
J G\left(u_{h, \delta}(t)\right) \leq \frac{3}{2}, \quad\left|J G\left(u_{h, \delta}(t)\right)-1\right| \leq \frac{2}{\omega_{\max }}\left\|v_{h, \lambda}\right\|_{0, \infty ; \mathcal{I}} \epsilon_{h}(t), \\
\left\|D G\left(u_{h, \delta}(t)\right)\right\| \leq c\left(\omega_{\max },\left\|v_{h, \lambda}\right\|_{1, \infty ; \mathcal{I}}\right) \\
\left\|E-D G\left(u_{h, \delta}(t)\right)\right\| \leq c\left(\omega_{\max },\left\|v_{h, \lambda}\right\|_{1, \infty ; \mathcal{I}}\right) \epsilon_{h}(t),
\end{aligned}
$$

where $\|\cdot\|$ is the matrix norm induced by $|\cdot|$. Combing this with the formulas (4.8) and (4.11) for $\theta_{h, \delta}^{1}$ yields the desired result that $R_{h, \delta}\left(\chi_{h}\right)$ in (4.10) is $O_{Z_{h}(t)}\left(\epsilon_{h}^{2} ; \chi_{h}\right)$. Taking into account also (4.9), we therefore showed that the discrete bulk equation (3.10c) holds up to terms of order $O_{Z_{h}(t)}\left(\epsilon_{h}^{2} ; \chi_{h}\right)$, iff

$$
\dot{\epsilon}_{h}=k\left[W_{h, \lambda}^{s} A_{h}^{s}-W_{h, \lambda}^{l} A_{h}^{l}\right] \epsilon_{h} .
$$

It remains to deal with the semidiscrete Gibbs-Thomson law (3.10d). Since

$$
\begin{aligned}
\beta \int_{\mathcal{I}} \frac{\partial_{t} u_{h, \delta}}{\sqrt{1+\left|D u_{h, \delta}\right|^{2}}} \varphi_{h} & =\beta \dot{\epsilon}_{h} \int_{\mathcal{I}} v_{h, \lambda} \varphi_{h}+O_{X_{h}}\left(\left|\dot{\epsilon}_{h} \epsilon_{h}\right| ; \varphi_{h}\right), \\
\gamma \int_{\mathcal{I}} \frac{D u_{h, \delta}}{\sqrt{1+\left|D u_{h, \delta}\right|^{2}}} D \varphi_{h} & =\lambda \gamma \epsilon_{h} \int_{\mathcal{I}} v_{h, \lambda} \varphi_{h}+O_{X_{h}}\left(\epsilon_{h}^{2} ; \varphi_{h}\right),
\end{aligned}
$$

and

$$
\lim _{y \nearrow u_{h, \delta}(x, t)} \theta_{h, \delta}(x, y, t)=-\beta \hat{V}+\epsilon_{h}(t)\left[p^{s}+A_{h}^{s}\right] v_{h, \lambda}(x),
$$

the semidiscrete Gibbs-Thomson law (3.10d), where the discrete temperature field $\theta_{h, \delta}$ is evaluated from the solid, holds up to $O_{X_{h}}\left(\epsilon_{h}^{2}+\left|\dot{\epsilon}_{h} \epsilon_{h}\right| ; \varphi_{h}\right)$, iff

$$
\beta \dot{\epsilon}_{h}=-\left[\lambda \gamma+p^{s}+A_{h}^{s}\right] \epsilon_{h} .
$$

Similarly, equation (3.10d), where the discrete temperature field $\theta_{h, \delta}$ is evaluated from the liquid, holds up $O_{X_{h}}\left(\epsilon_{h}^{2}+\left|\dot{\epsilon}_{h} \epsilon_{h}\right| ; \varphi_{h}\right)$, iff

$$
\beta \dot{\epsilon}_{h}=-\left[\lambda \gamma+p^{l}+A_{h}^{l}\right] \epsilon_{h}
$$

The system (4.13) and (4.14), which has the same structure as (2.9) and (2.11), is solved uniquely by

$$
\begin{aligned}
A_{h}^{s} & =-\frac{\gamma \lambda+p^{s}-\beta W_{h, \lambda}^{l} \hat{V}}{1+\beta k\left(W_{h, \lambda}^{s}-W_{h, \lambda}^{l}\right)}, \\
A_{h}^{l} & =-\frac{\gamma \lambda+p^{l}-\beta W_{h, \lambda}^{s} \hat{V}}{1+\beta k\left(W_{h, \lambda}^{s}-W_{h, \lambda}^{l}\right)}, \\
\epsilon_{h}(t) & =\delta \mathrm{e}^{\mu_{h} t}
\end{aligned}
$$

with

$$
\mu_{h}:=k \frac{\left(W_{h, \lambda}^{s}-W_{h, \lambda}^{l}\right)(-\gamma \lambda)+W_{h, \lambda}^{l} p^{l}-W_{h, \lambda}^{s} p^{s}}{1+\beta k\left(W_{h, \lambda}^{s}-W_{h, \lambda}^{l}\right)}
$$


for all $t \in[0, T$. These quantities are well-defined because of (4.3). Moreover, recalling (2.4), we observe that (4.15a) and (4.15b) are consistent with (4.6) and so condition (3.10b) holds provided (3.10a). Therefore, we have proved the following semidiscrete counterpart of Theorem 2.1.

Theorem 4.1 (Approximate semidiscrete solutions). Fix an eigenvalue $\lambda \in \Lambda_{h}$ of $-D_{h}^{2}$ with (discrete) natural boundary conditions, let $v_{h, \lambda}$ be a corresponding eigenfunction, and suppose that (2.5) holds.

Then ansatz (4.1), (4.5) with (4.15) provides a family $\left(\theta_{h, \delta}, u_{h, \delta}\right)_{\delta}$ such that

- if $|\delta|$ is sufficiently small, then $\theta_{h, \delta}$ and $u_{h, \delta}$ satisfy (3.10a), (3.10b), and (3.10e) with $u_{h, \text { init }}=\delta v_{h, \lambda}$.

- for $\delta \rightarrow 0$, the functions $\theta_{h, \delta}$ and $u_{h, \delta}$ fulfill

$$
\int_{\Omega} k \nabla \theta_{h, \delta} \cdot \nabla \chi_{h}=\int_{\mathcal{I}}\left(\frac{\partial_{t} u_{h, \delta}}{\sqrt{1+\left|D u_{h, \delta}\right|^{2}}}+\hat{V}\right) \chi_{h}\left(u_{h, \delta}\right) \sqrt{1+\left|D u_{h, \delta}\right|^{2}}+O_{Z_{h}(t)}\left(\delta^{2} ; \chi_{h}\right)
$$

and

$$
\beta \int_{\mathcal{I}} \frac{\partial_{t} u_{h, \delta}}{\sqrt{1+\left|D u_{h, \delta}\right|^{2}}} \varphi_{h}+\gamma \int_{\mathcal{I}} \frac{D u_{h, \delta}}{\sqrt{1+\left|D u_{h, \delta}\right|^{2}}} D \varphi_{h}=-\int_{\mathcal{I}}\left[\theta_{h}\left(u_{h, \delta}\right)+\beta \hat{V}\right] \varphi_{h}+O_{X_{h}}\left(\delta^{2} ; \varphi_{h}\right)
$$

for all $\chi_{h} \in Z_{h}(t):=Z_{h}\left(u_{h, \delta}(t)\right), \varphi_{h} \in X_{h}$, and $t \in\left[0, T\left[\right.\right.$. Here $O_{V}\left(\delta^{2} ; \cdot\right)$ indicates a linear functional on $V$ whose norm, see (4.7), is bounded by $\delta^{2}$ times a constant depending on data, $\lambda$, the choice of $v_{h, \lambda}$, and the discrete spaces $X_{h}$ and $Y_{h}$.

As in the continuous case, one expects that the amplification rate $\mu_{h}$ in (4.15d) indicates the stability of the corresponding discrete mode. It is worthwhile to mention that this will be confirmed in Section 6; compare Corollary 6.1 and Remark 6.1.

\section{Comparison And Discussion}

In this section we compare and illustrate the results obtained in the continuous (Sect. 2) and in the semidiscrete (Sect. 4) case. We then discuss consequences on discretization issues. Finally, supposing that the results essentially carry over to discretizations of the modified Stefan problem (1.1), we comment on the two motivating issues of the introduction.

\subsection{Comparing $\mu_{h}$ with $\mu-$ part 1}

We start with some theoretical considerations concerning the formulae (2.12d) and (4.15d) for the amplification rates $\mu$ and $\mu_{h}$, respectively. For the sake of simplicity, we restrict ourselves to the case that the partition of $\mathcal{J}$ is symmetric with respect to 0 , i.e. $\underline{J}=-\bar{J}$ and $y_{-j}=y_{j}$ for $j=1, \ldots, \bar{J}$. Then $W_{h, \lambda}^{s}=-W_{h, \lambda}^{l}$ and so (4.15d) simplifies to

$$
\mu_{h}=\frac{k W_{h, \lambda}}{1+2 \beta k W_{h, \lambda}}\left(-2 \gamma \lambda-p^{l}-p^{s}\right)
$$

with $W_{h, \lambda}:=W_{h, \lambda}^{s}$. This corresponds to (2.12d) apart from the replacement of $W_{\lambda}$ by $W_{h, \lambda}$ and the fact that $\lambda$ is taken from $\Lambda_{h}$ instead of $\Lambda$. In particular, $\mu_{h}$ and $\mu$ - interpreted as functions of $\lambda \in \mathbb{R}_{0}^{+}$- change sign in the same point. Note also that the sign of $\mu_{h}$ in (5.1) depends only (through $\lambda$ ) on $X_{h}$, while its absolute value depends on both discrete spaces $X_{h}$ and $Y_{h}$.

In what follows, it is convenient to number the elements of the spectra $\Lambda$ and $\Lambda_{h}$ according to their size:

$$
0 \leq \lambda_{0} \leq \cdots \leq \lambda_{m} \leq \cdots \quad \text { and } \quad 0 \leq \lambda_{h, 0} \leq \cdots \leq \lambda_{h, I},
$$


respectively. Evaluating $(2.12 \mathrm{~d})$ and (5.1) for a fixed data set then yields the sequences $\left(\mu_{m}\right)_{m \in \mathbb{N}_{0}}$ and $\left(\mu_{h, m}\right)_{m=0}^{I}$, respectively. Let $m \in\{0, \ldots, I\}$. The Minmax principle yields

$$
\lambda_{m} \leq \lambda_{h, m}
$$

compare with e.g. [17] ((23), Chap. 6). Therefore, the monotonicity of the function $\mathbb{R}_{0}^{+} \ni \lambda \mapsto W_{\lambda}$, the identity in (4.3a), and (2.10) imply

$$
W_{h, \lambda_{h, m}} \geq W_{\lambda_{m}}
$$

Consequently, we have the following: if $\mu_{m} \leq 0$, then $-2 \gamma \lambda_{m}-p^{l}-p^{s} \leq 0$ and so $-2 \gamma \lambda_{h, m}-p^{l}-p^{s} \leq 0$ as well as

$$
\mu_{h, m} \leq \mu_{m} \leq 0
$$

In other words: if a continuous mode is "stable", then its semidiscrete counterpart (if it exists) is also stable and decays faster.

It remains to discuss the effect of the spatial discretization for continuous modes with

$$
\lambda_{m} \leq \lambda_{\text {crit }}:=-\frac{p^{l}+p^{s}}{2 \gamma}
$$

\subsection{Comparing $\mu_{h}$ with $\mu-$ part 2}

If a continuous mode is "unstable", that is $\mu_{m}>0$, then a comparison of $\mu_{m}$ and $\mu_{h, m}$ is more delicate: the increasing of the quotient in (5.1) may be compensated by the decreasing of the term between the parentheses. Alternatively, we illustrate what happens with an example, where $\mu_{m}$ and $\mu_{h, m}$ are computed numerically. To this end, we fix data to be

$$
k=1, \quad \beta=0.01, \quad \gamma=5 \cdot 10^{-5}, \quad \theta^{l}=-0.5, \quad \theta^{s}=0,
$$

which implies that the liquid is undercooled and all the latent heat flows into it.

We start by visualizing the relationship between $\lambda_{m}$ and $\mu_{m}$. Figure 1 (left-hand side) depicts $\mu_{m}$ versus $\lambda_{m}$ for $0 \leq m \leq 150$. We observe that the most "unstable" continuous modes are around $\lambda=1332.25$ with maximal amplification rate $\mu_{\max } \approx 9.8$.

Next, we analyze the relationship between $\mu_{h, m}$ and $\lambda_{h, m}$ for various choices of $X_{h}$ and $Y_{h}$.

Linear elements $(q=r=1)$ on uniform partitions with step size $h_{\mathcal{I}} \approx 0.042$ (that is $\left.\operatorname{dim} X_{h}=151\right)$ and $h_{\mathcal{J}}=0.01$ have been used for the right-hand side of Figure 1 . We observe that the "unstable" continuous modes close to $\lambda=5000$ have stable discrete counterparts. Moreover, similarly to the continuous case, the most unstable discrete modes are around $\lambda=1360.64$ with maximal discrete amplification rate $\mu_{\max } \approx 9.85$.

It is instructive to consider the effect of coarsening (enlarging the meshsize) of $X_{h}$ and of $Y_{h}$ separately.

We first consider the effect of a relatively coarse $X_{h}$, that is the relatively coarse spatial discretization on and parallel to the interface. In the left-hand side of Figure 2 we used the same data as in the right-hand side of Figure 1 apart from $h_{\mathcal{I}} \approx 0.085$ (that is $\operatorname{dim} X_{h}=75$ ) instead of $h_{\mathcal{I}} \approx 0.042$. Remarkably, all discrete modes are unstable in the coarse case. This is consistent with the following theoretical consideration: The inverse estimate $\left\|D \varphi_{h}\right\|_{0,2 ; \mathcal{I}} \leq C_{q} h_{\mathcal{I}}^{-1}\left\|\varphi_{h}\right\|_{0,2 ; \mathcal{I}}$ implies

$$
\lambda=\frac{\left\|D v_{h, \lambda}\right\|_{0,2 ; \mathcal{I}}^{2}}{\left\|v_{h, \lambda}\right\|_{0,2 ; \mathcal{I}}^{2}} \leq C_{q}^{2} h_{\mathcal{I}}^{-2}
$$

for any $\lambda \in \Lambda_{h}$, where the constant $C_{q}$ depends only on the polynomial degree $q$ of the discrete space $X_{h}$. Consequently, if $h_{\mathcal{I}}>C_{q} \sqrt{-2 \gamma /\left(p^{l}+p^{s}\right)}$, then $\mu_{h}>0$ for all $\lambda \in \Lambda_{h}$. In other words: if $h_{\mathcal{I}}$ is not small with 

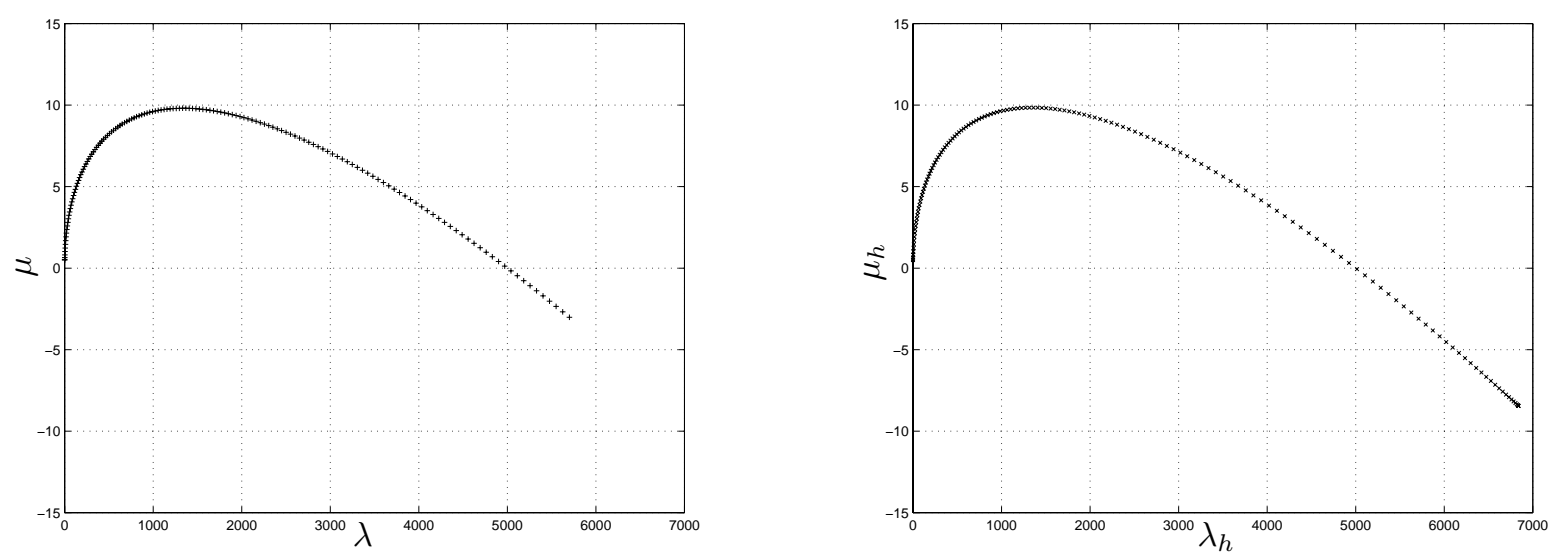

FIgURE 1. Stability of continuous and semidiscrete modes for data (5.2).
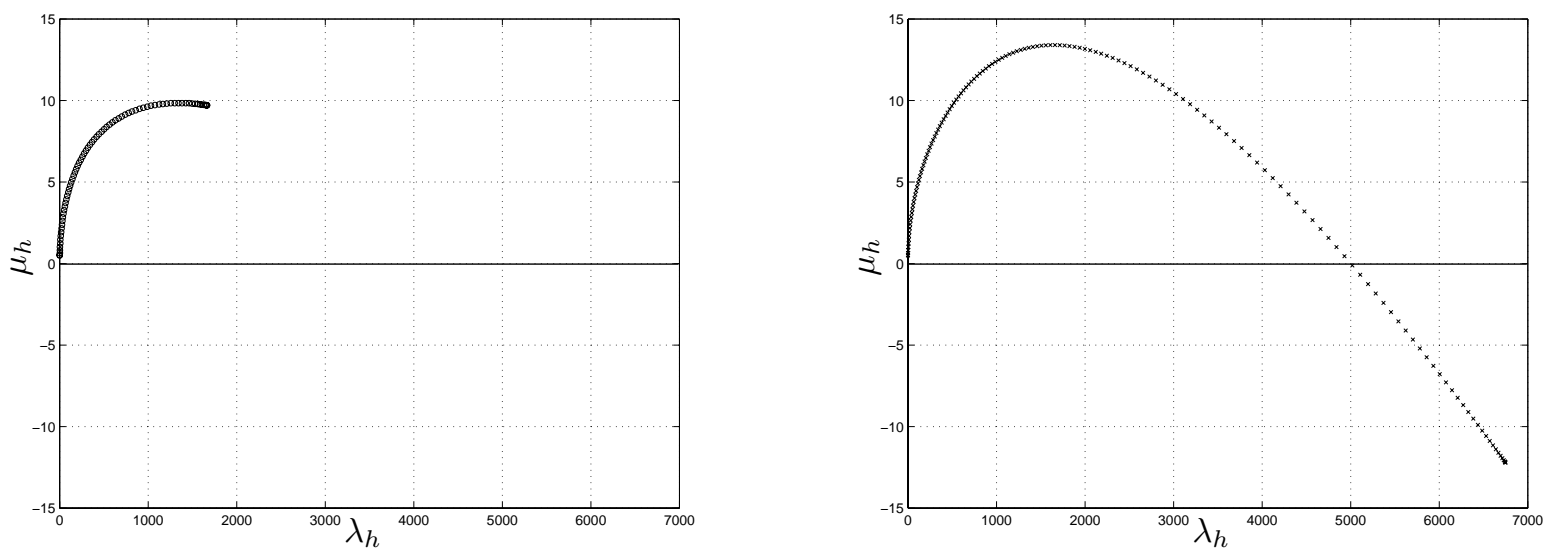

Figure 2. The effect of coarse $X_{h}$ and coarse $Y_{h}$ for data (5.2).

respect to the quotient of surface tension and "effective" undercooling or superheating, then all discrete modes are unstable.

We next discuss the effect of a relatively coarse $Y_{h}$, that is a relatively coarse spatial discretization orthogonal to the interface. We compare again with the right-hand side of Figure 1 and replace $h_{\mathcal{J}}=0.01$ by $h_{\mathcal{J}}=0.1$ in the right-hand side of Figure 2. As expected, $Y_{h}$ has no effect on the sign of the discrete amplification rate $\mu_{h}$, that is the stability of the discrete mode. However, the absolute value of $\mu_{h}$, that is how fast a discrete mode grows or decays, depends on $Y_{h}$. For the coarse $Y_{h}$, the most unstable modes are around $\lambda=1660.54$ with $\mu_{h, \max } \approx 12.9$. This faster growth is consistent with (4.4) and the fact that $W_{h, \lambda}=W_{h, \lambda}^{s}$ enters in (5.1) in a monotone way.

One may wonder if higher order polynomials, i.e. $q>1$ or $r>1$, in the definition of $X_{h}$ and $Y_{h}$ lead to better results. Their use for $X_{h}$ does not offer a meaningful improvement for the same dimension of $X_{h}$; compare the left-hand side of Figure 2 with the left-hand side of Figure 3, where $X_{h}$ is the space of continuous quadratic elements with step size $h_{\mathcal{I}} \approx 0.17$. However, the fact that (4.4) is a discretization of the smooth minimization (2.10) suggests that the use of higher order polynomials for $Y_{h}$ is advantageous. In fact, the right-hand sides of Figures 2 and 3 reveal that, for the same dimension of $Y_{h}$, quadratic elements lead to better stabilities properties that linear ones: in the case of Figure 3 (right-hand side) the most unstable modes are around $\lambda=1660.54$ with $\mu_{h, \max } \approx 11.8$. 

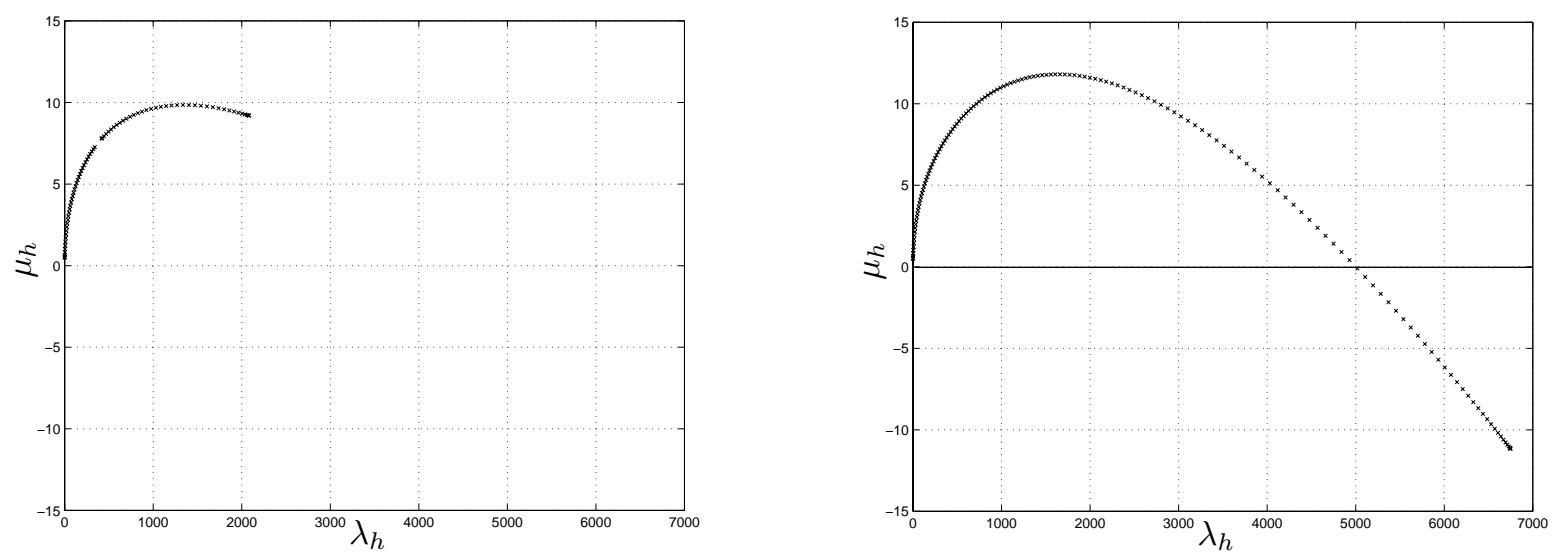

Figure 3 . The effect of quadratic $X_{h}$ and quadratic $Y_{h}$ for data (5.2).

We may summarize as follows: If the spatial discretization along the interface does not contain enough degrees of freedom, the semidiscrete Problem 3.1 is "completely unstable" (while the continuous Problem 2.1 has many stable modes). If the spatial discretization orthogonal to the interface is not fine enough (regarding grid and ansatz spaces), the most unstable modes of the semidiscrete Problem 3.1 may grow much faster that those of the continuous Problem 2.1.

\subsection{Consequences}

We continue by discussing consequences on discretization issues of the preceding results.

A first consequence concerns the discretization $u_{h \text {,init }} \in X_{h}$ of the initial value $u_{\text {init }}$. To simplify the discussion, let us neglect other discretization effects by supposing momentarily that $u_{h}$ is the exact solution of the continuous problem, where the initial value $u_{\text {init }}$ is replaced by $u_{h \text {,init }}$. Then Section 2 suggests that the choice of $u_{h \text {,init }}$ is a delicate matter: in order to obtain a "discrete" solution $u_{h}$ that is meaningful with respect to the original initial value $u_{\text {init }}$, the error $u_{h \text {,init }}-u_{\text {init }}$ should have quite small contributions of unstable modes; in the case of Figure 1 (left-hand side) one has $\epsilon(2) \approx 32$ for $\lambda=1332.25$ and $\delta=10^{-7}$. In general, this requires a certain fineness of the discrete space $X_{h}$ and an appropriate choice therein.

Even for relatively fine choices of $X_{h}$ and $Y_{h}$ a situation as in the right part of Figure 1 (right-hand side) can occur. Consequently, the approximate solution of the semidiscrete Problem 3.1 has to be done with care such that the errors in unstable modes are quite small. For otherwise, "unnecessary" instabilities may develop. This is especially important, if $Y_{h}$ is relatively coarse: in the case of Figure 2 (right-hand side) one has $\epsilon_{h}(1.5) \approx 25$ for $\lambda=1660.54$ and $\delta=10^{-7}$.

We recommend - regardless of the resulting convergence speed (which may be affected by additional nonregular source terms) - higher order polynomials for $Y_{h}$, because they lead to better stability properties.

\subsection{Further remarks}

The above stability analyses seem to capture the essential aspects of the coupling of the Stefan condition (1.1b) and the Gibbs-Thomson law (1.1c). One may therefore suppose that the obtained results essentially carry over to unperturbed solutions differing from flat interfaces and to similar models as the modified Stefan problem (1.1). This is supported by the following facts:

- Continuous stability analyses for growing spherical interfaces yield similar results, see e.g. [11] (Sect. III.B).

- Also flat interfaces of the modified Stefan problem (1.1) exhibit similar stability properties; see the more involved analysis [16] by Strain who reformulates (1.1) in terms of a history-dependent singular integral equation for the normal velocity $V$ of the interface. 
The observation concerning the jump of the discrete heat flow (see the end of Section 3) suggests that the stability properties of other (semi)discretizations, e.g. those in $[3,14,18]$, are less close to the continuous stability properties.

Keeping this in mind, an explanation of the secondary side branching observed in Schmidt [14] (Fig. 4.5) as numerical instabilities seems to be reasonable. However, in order to decide whether or not the continuous model exhibits secondary side branching, one should increase the simulation time $T$, since the "continuous" secondary side branching may appear at a later time. Moreover, one expects the constant $C$ in (1.2) of moderate size, only if the meshsize is small with respect to the quotient of surface tension and "effective" undercooling or superheating. Finally, a dependence of $C$ on $T$ is indicated by the fact that the continuous "unstable" modes are not resolved exactly in the discrete spaces in general.

\section{Classical stability for Semidiscrete flat interfaces}

In this section we show that the analysis for the semidiscrete Problem 3.1 in Section 4 corresponds to a classical (linearized) stability analysis of flat interfaces. This correspondence is established in two steps: first, taking advantage of $\beta>0$, we rewrite the semidiscrete Problem 3.1 as an initial value problem of the form:

$$
\dot{U}=\mathcal{F}(U) \text { in }] 0, T\left[, \quad U(0)=U^{0} .\right.
$$

Hereafter, $U=\left(U_{0}, \ldots, U_{I}\right):\left[0, T\left[\rightarrow \mathbb{R}^{I+1}\right.\right.$ is the time-dependent coefficient vector defined by

$$
u_{h}(t)=\sum_{m=0}^{I} U_{m}(t) \varphi_{m}
$$

for all $t \in\left[0, T\right.$. Second, we calculate the eigenvalues of $\mathcal{F}^{\prime}(0)$ in terms of data and the eigenvalues of the operator $-D_{h}^{2}$. As a side effect of the reformulation (6.1), we also obtain existence and uniqueness of the semidiscrete Problem 3.1 for sufficiently small $T>0$.

In order to reformulate the semidiscrete Problem 3.1, we fix partitions of $\mathcal{I}$ and $\mathcal{J}$ and use the notation introduced in Section 3. For $W=\left(W_{0}, \ldots, W_{I}\right) \in \mathbb{R}^{I+1}$, we set

$$
\begin{aligned}
Q(W) & :=\left(1+\left|\sum_{i=0}^{I} W_{i} D \varphi_{i}\right|^{2}\right)^{1 / 2}, \\
\mathcal{M}^{\mathcal{I}}(W) & :=\left(\mathcal{M}_{i, m}^{\mathcal{I}}(W)\right)_{i, m=0, \ldots, I} \quad \text { with } \quad \mathcal{M}_{i, m}^{\mathcal{I}}(W):=\beta \int_{\mathcal{I}} \frac{\varphi_{m}}{Q(W)} \varphi_{i}, \\
\mathcal{S}^{\mathcal{I}}(W) & :=\left(\mathcal{S}_{i, m}^{\mathcal{I}}(W)\right)_{i, m=0, \ldots, I} \quad \text { with } \quad \mathcal{S}_{i, m}^{\mathcal{I}}(W):=\gamma \int_{\mathcal{I}} \frac{D \varphi_{m}}{Q(W)} D \varphi_{i}, \\
\mathcal{R}^{\mathcal{I}}(W) & :=\left(\mathcal{R}_{i}^{\mathcal{I}}(W)\right)_{i=0, \ldots, I} \quad \text { with } \quad \mathcal{R}_{i}^{\mathcal{I}}(W):=-\int_{\mathcal{I}}\left[\theta_{b}\left(\sum_{m=0}^{I} W_{m} \varphi_{m}\right)+\beta \hat{V}\right] \varphi_{i} .
\end{aligned}
$$

Moreover, we introduce the time-dependent coefficient vector $\Theta=\left(\Theta_{m n}\right)_{m n}:\left[0, T\left[\rightarrow \mathbb{R}^{(I+1) J}, J:=-\underline{J}+\bar{J}+1\right.\right.$, by the identity

$$
\theta_{h}(t)-\theta_{b}=\sum_{m=0}^{I} \sum_{n=\underline{J}}^{\bar{J}} \Theta_{m n}(t) \hat{\chi}_{m n} \circ G\left(u_{h}(t)\right)^{-1}
$$

for all $t \in\left[0, T\left[\right.\right.$. Here, we used the following convention: $\Theta_{m n}(t)$ denotes the $(m(J+1)+n-\underline{J})^{\text {th }}$ component of the vector $\Theta(t)$, while, for example, $\mathcal{M}_{i, m}^{\mathcal{I}}(W)$ indicates the entry in the $i^{\text {th }}$ row and $m^{\text {th }}$ column of the 
matrix $\mathcal{M}^{\mathcal{I}}(W)$. In addition, we define

$$
\mathcal{M}^{\mathcal{I}}:=\mathcal{M}^{\mathcal{I}}(0), \quad \mathcal{P}:=\left(\mathcal{P}_{i, m n}\right)_{i, m=0, \ldots, I ; n=\underline{J}, \ldots, \bar{J}} \text { with } \mathcal{P}_{i, m n}:=\delta_{i, m} \delta_{n, 0}
$$

Supposing (6.2) and (6.3), the discrete Gibbs-Thomson law (3.10d) is equivalent with

$$
\mathcal{M}^{\mathcal{I}}(U) \dot{U}+\mathcal{S}^{\mathcal{I}}(U) U=-\mathcal{M}^{\mathcal{I}} \mathcal{P} \Theta+\mathcal{R}^{\mathcal{I}}(U)
$$

in $] 0, T[$.

Next, we give an analogue equivalence for the discrete bulk equation (3.10c). To this end, we define

$$
\mathcal{B}:=\left\{W=\left(W_{0}, \ldots, W_{I}\right) \in \mathbb{R}^{I+1} \mid \sum_{i=0}^{I} W_{i} \varphi_{i} \in B_{h}\right\}
$$

and, for $W=\left(W_{0}, \ldots, W_{I}\right) \in \mathcal{B}$,

$$
\begin{aligned}
\mathcal{G}^{-1}(W):= & G^{-1}\left(\sum_{i=0}^{I} W_{i} \varphi_{i}\right), \\
\mathcal{S}^{\Omega}(W):= & \left(\mathcal{S}_{i j, m n}^{\Omega}(W)\right)_{i, m=0, \ldots, I ; j, n=\underline{J}, \ldots, \bar{J}} \\
& \quad \text { with } \mathcal{S}_{i j, m n}^{\Omega}(W):=\int_{\Omega} \nabla\left[\hat{\chi}_{m n} \circ \mathcal{G}^{-1}(W)\right] \cdot \nabla\left[\hat{\chi}_{i j} \circ \mathcal{G}^{-1}(W)\right], \\
\mathcal{R}^{\Omega}(W):= & \left(\mathcal{R}_{i j}^{\Omega}(W)\right)_{i=0, \ldots, I ; j=\underline{J}, \ldots, \bar{J}} \\
& \quad \text { with } \mathcal{R}_{i j}^{\Omega}(W):=\int_{\mathcal{I}} \hat{V} \varphi_{i} \delta_{j, 0} Q(W)-\int_{\Omega} \nabla \theta_{b} \cdot \nabla\left[\hat{\chi}_{i j} \circ \mathcal{G}^{-1}(W)\right] .
\end{aligned}
$$

Then, again supposing (6.2) and (6.3), condition (3.10a) is equivalent to

$$
U(t) \in \mathcal{B}
$$

for all $t \in] 0, T[$ and the discrete bulk equation (3.10c) is equivalent to

$$
\mathcal{S}^{\Omega}(U) \Theta=\mathcal{P}^{T} \mathcal{M}^{\mathcal{I}} \dot{U}+\mathcal{R}^{\Omega}(U)
$$

in $] 0, T$ [, where $\mathcal{P}^{T}$ is the transpose of $\mathcal{P}$.

It remains to "decouple" the system (6.4), (6.6). We first observe that $\mathcal{M}^{\mathcal{I}}(U)$ is symmetric, positive definite (recall $\beta>0$ ) and thus invertible. So we may write

$$
\dot{U}=-\mathcal{M}^{\mathcal{I}}(U)^{-1}\left[\mathcal{S}^{\mathcal{I}}(U) U+\mathcal{M}^{\mathcal{I}} \mathcal{P} \Theta-\mathcal{R}^{\mathcal{I}}(U)\right]
$$

and use this in (6.6) to obtain

$$
\left[\mathcal{S}^{\Omega}(U)+\mathcal{Z}(U)\right] \Theta=-\mathcal{P}^{T} \mathcal{M}^{\mathcal{I}} \mathcal{M}^{\mathcal{I}}(U)^{-1}\left[\mathcal{S}^{\mathcal{I}}(U) U-\mathcal{R}^{\mathcal{I}}(U)\right]+\mathcal{R}^{\Omega}(U)
$$

with

$$
\mathcal{Z}(W):=\mathcal{P}^{T} \mathcal{M}^{\mathcal{I}} \mathcal{M}^{\mathcal{I}}(W)^{-1} \mathcal{M}^{\mathcal{I}} \mathcal{P}
$$

for all $W \in \mathcal{B}$. Both matrices $\mathcal{S}^{\Omega}(W)$ and $\mathcal{Z}(W)$ are symmetric. Moreover, $\mathcal{S}^{\Omega}(W)$ is positive definite and 
$\mathcal{Z}(W)$ is positive semidefinite. Consequently, $\left[\mathcal{S}^{\Omega}(U)+\mathcal{Z}(U)\right]^{-1}$ exists and we may write

$$
\begin{aligned}
\Theta & =\left[\mathcal{S}^{\Omega}(U)+\mathcal{Z}(U)\right]^{-1}\left(-\mathcal{P}^{T} \mathcal{M}^{\mathcal{I}} \mathcal{M}^{\mathcal{I}}(U)^{-1}\left[\mathcal{S}^{\mathcal{I}}(U) U-\mathcal{R}^{\mathcal{I}}(U)\right]+\mathcal{R}^{\Omega}(U)\right), \\
\dot{U} & =\mathcal{F}(U),
\end{aligned}
$$

where, for $W \in \mathcal{B}$,

$$
\begin{aligned}
& \mathcal{F}(W):=-\mathcal{M}^{\mathcal{I}}(W)^{-1}\left(E-\mathcal{M}^{\mathcal{I}} \mathcal{P}\left[\mathcal{S}^{\Omega}(W)+\mathcal{Z}(W)\right]^{-1} \mathcal{P}^{T} \mathcal{M}^{\mathcal{I}} \mathcal{M}^{\mathcal{I}}(W)^{-1}\right) \\
& \cdot\left[\mathcal{S}^{\mathcal{I}}(W) W-\mathcal{R}^{\mathcal{I}}(W)\right]+\mathcal{M}^{\mathcal{I}}(W)^{-1} \mathcal{M}^{\mathcal{I}} \mathcal{P}\left[\mathcal{S}^{\Omega}(W)+\mathcal{Z}(W)\right]^{-1} \mathcal{R}^{\Omega}(W)
\end{aligned}
$$

We summarize:

Lemma 6.1 (Reformulation as ODE). Suppose that (6.2), (6.3), and

$$
u_{h, \text { init }}=\sum_{m=0}^{I} U_{0}^{m} \varphi_{m}
$$

hold. Then $u_{h}$ and $\theta_{h}$ solve the semidiscrete Problem 3.1, if $U$ and $\Theta$ satisfy (6.5), the decoupled equations (6.7), and $U(0)=\left(U_{0}^{0}, \ldots, U_{0}^{I}\right)$.

Since $\mathcal{F}: \mathcal{B} \rightarrow \mathbb{R}^{I+1}$ is continuously differentiable, the theorem of Picard-Lindelöf, see e.g. [2, (7.6)], ensures that $(6.7 \mathrm{~b})$ has a unique solution for some time.

Theorem 6.1 (Existence and uniqueness of semidiscrete solutions). Suppose that $u_{h, i n i t} \in B_{h}$. Then the semidiscrete Problem 3.1 admits a unique solution on $[0, T[$, if $T>0$ is sufficiently small.

A flat interface that moves with velocity $\hat{V}$ satisfying (2.5) along the $y$-axis corresponds to the stationary solution $U(t) \equiv 0$ of $(6.7 \mathrm{~b})$. In order to perform a classical linearized stability analysis of such flat interface, we thus have to determine the eigenvalues of $\mathcal{F}^{\prime}(0)$, the Jacobian matrix of $\mathcal{F}$ in $0 \in \mathbb{R}^{I+1}$. Theorem 4.1 implies:

Corollary 6.1 (Linearized stability analysis). Suppose that (2.5) holds. Then the matrix $\mathcal{F}^{\prime}(0)$ has the same eigenvectors as the generalized eigenvalue problem

$$
\mathcal{S}^{\mathcal{I}} V=\lambda \mathcal{M}^{\mathcal{I}} V
$$

where $\mathcal{S}^{\mathcal{I}}:=\mathcal{S}^{\mathcal{I}}(0)$ and $\mathcal{M}^{\mathcal{I}}$ are the stiffness and mass matrix belonging to the partition of $\mathcal{I}$, respectively. Moreover, the eigenvalue of $\mathcal{F}^{\prime}(0)$ corresponding to the eigenvalue $\lambda$ of (6.8) (or the operator $-D_{h}^{2}$ with discrete natural boundary conditions) is

$$
k \frac{\left(W_{h, \lambda}^{s}-W_{h, \lambda}^{l}\right)(-\gamma \lambda)+W_{h, \lambda}^{l} p^{l}-W_{h, \lambda}^{s} p^{s}}{1+\beta k\left(W_{h, \lambda}^{s}-W_{h, \lambda}^{l}\right)} .
$$

Proof. Let $\left(\lambda, V^{\lambda}\right)$ with $V^{\lambda}=\left(V_{0}^{\lambda}, \ldots, V_{I}^{\lambda}\right) \in \mathbb{R}^{I+1}$ be an eigenpair of (6.8). Then, $\left(\lambda, v_{h, \lambda}\right)$ with $v_{h, \lambda}:=$ $\sum_{m=0}^{I} V_{m}^{\lambda} \varphi_{m}$ is an eigenpair of $-D_{h}^{2}$ with discrete homogeneous Neumann boundary conditions. For $T>0$ fixed and $\delta$ sufficiently close to 0 , let $\left(\theta_{h, \delta}, u_{h, \delta}\right)$ be the corresponding approximate solution of the semidiscrete Problem 3.1 defined by $(4.1,4.5)$, and (4.15). Moreover, define $U^{\delta}=\left(U_{0}^{\delta}, \ldots, U_{I}^{\delta}\right):\left[0, T\left[\rightarrow \mathbb{R}^{I+1}\right.\right.$ and $\Theta^{\delta}=\left(\Theta_{m n}^{\delta}\right)_{m n}:\left[0, T\left[\rightarrow \mathbb{R}^{(I+1) J}\right.\right.$ by the identities

$$
u_{h, \delta}=\sum_{m=0}^{I} U_{m}^{\delta} \varphi_{m}, \quad \theta_{h, \delta}-\theta_{b}=\sum_{m=0}^{I} \sum_{n=\underline{J}}^{\bar{J}} \Theta_{m n}^{\delta} \hat{\chi}_{m n} \circ G\left(u_{h, \delta}\right)^{-1}
$$


We test (3.10c) and (3.10d) with the basis function of $X_{h}$ and $Z_{h}\left(G\left(u_{h, \delta}(t)\right)\right)$ and, upon taking into account (4.12), obtain

$$
\begin{aligned}
\mathcal{M}^{\mathcal{I}}\left(U^{\delta}\right) \dot{U}^{\delta}+\mathcal{S}^{\mathcal{I}}\left(U^{\delta}\right) U^{\delta} & =-\mathcal{M}^{\mathcal{I}} \mathcal{P} \Theta^{\delta}+\mathcal{R}^{\mathcal{I}}\left(U^{\delta}\right)+O\left(\delta^{2}\right) \\
\mathcal{S}^{\Omega}\left(U^{\delta}\right) \Theta^{\delta} & =\mathcal{P}^{T} \mathcal{M}^{\mathcal{I}} \dot{U}^{\delta}+\mathcal{R}^{\Omega}\left(U^{\delta}\right)+O\left(\delta^{2}\right)
\end{aligned}
$$

in $] 0, T[$ as $\delta \rightarrow 0$. Note that this is also true in $[0, T[$. We can thus derive

$$
\dot{U}^{\delta}=\mathcal{F}\left(U^{\delta}\right)+O\left(\delta^{2}\right)
$$

in $\left[0, T\left[\right.\right.$ by following the calculations preceding Lemma 6.1. Using $\mathcal{F}(0)=0,(6.10)$ in $t=0$, and $U^{\delta}(t)=$ $\delta \mathrm{e}^{\mu_{h} t} V^{\lambda}$, we calculate

$$
\mathcal{F}^{\prime}(0) V^{\lambda}=\lim _{\delta \rightarrow 0} \frac{\mathcal{F}\left(\delta V^{\lambda}\right)-\mathcal{F}(0)}{\delta}=\lim _{\delta \rightarrow 0} \frac{\dot{U}^{\delta}(0)+O\left(\delta^{2}\right)}{\delta}=\mu_{h} V^{\lambda},
$$

which finishes the proof.

Remark 6.1 (Nonlinear stability analysis). Suppose that expression (6.9) is nonzero for all $\lambda \in \Lambda_{h}$, where $\Lambda_{h}$ is the spectrum of (6.8) (or $-D_{h}^{2}$ with (discrete) natural boundary conditions). In this generic case the fixed point $0 \in \mathbb{R}^{I+1}$ is hyperbolic and the theorem of Grobman and Hartman, see e.g. [13] (Sect. 2.8), implies that the response of $(6.7 \mathrm{~b})$ on small perturbations of the initial value 0 is essentially described by $\dot{U}=\mathcal{F}^{\prime}(0) U$.

Corollary 6.1 and Remark 6.1 imply that the stability of "hyperbolic" semidiscrete flat interfaces can be investigated by means of the approximate solutions in Theorem 4.1. In particular, we obtain that hyperbolic flat semidiscrete interfaces whose latent heat completely flows into the undercooled liquid are unstable. Moreover, the two aforementioned results strongly underline the relevance of the discussion in Section 5.

Acknowledgements. This research was supported by the Deutsche Forschungsgemeinschaft through the project "Effiziente Simulation und Numerische Analysis der Dynamik von Dendriten" of the Schwerpunkt "Dynamik: Analysis, effiziente Simulation und Ergodentheorie". The author would like to thank G. Dziuk and A. Schmidt, who directed the aforementioned project, for interesting discussions on the topic of this article. The computations in Section 5 were carried out with the help of Matlab ${ }^{\circledR}$.

\section{REFERENCES}

[1] V. Alexiades and A.D. Solomon, Mathematical modeling of melting and freezing processes. Hemisphere Publishing Corporation, Washington (1993).

[2] H. Amann, Ordinary differential equations. An introduction to nonlinear analysis, Vol. 13 of De Gruyter Studies in Mathematics. Walter de Gruyter, Berlin (1990).

[3] E. Bänsch and A. Schmidt, A finite element method for dendritic growth, in Computational crystal growers workshop, J.E. Taylor Ed., AMS Selected Lectures in Mathematics (1992) 16-20.

[4] X. Chen, J. Hong and F. Yi, Existence, uniqueness, and regularity of classical solutions of the Mullins-Sekerka problem. Comm. Partial Differential Equations 21 (1996) 1705-1727.

[5] K. Deckelnick and G. Dziuk, Convergence of a finite element method for non-parametric mean curvature flow. Numer. Math. 72 (1995) 197-222.

[6] J. Escher and G. Simonett, Classical solutions for Hele-Shaw models with surface tension. Adv. Differential Equations 2 (1997) 619-642.

[7] J. Escher and G. Simonett, Classical solutions for the quasi-stationary Stefan problem with surface tension, in Papers associated with the international conference on partial differential equations, Potsdam, Germany, June 29-July 2, 1996, M. Demuth et al. Eds., Vol. 100. Akademie Verlag, Math. Res., Berlin (1997) 98-104. 
[8] L.C. Evans and R. Gariepy, Measure Theory and Fine Properties of Functions. CRC Press, Inc., 2000 Corporate Blvd., N.W., Boca Ratin, Stud. Adv. Math., 33431, Florida (1992).

[9] M. Fried, A level set based finite element algorithm for the simulation of dendritic growth. Submitted to Computing and Visualization in Science, Springer.

[10] M.E. Gurtin, Thermomechanics of evolving phase boundaries in the plane. Clarendon Press, Oxford (1993).

[11] J.S. Langer, Instabilities and pattern formation in crystal growth. Rev. Modern Phys. 52 (1980) 1-28.

[12] W.W. Mullins and R.F. Sekerka, Stability of a planar interface during solidification of a dilute binary alloy. J. Appl. Phys. 35 (1964) 444-451.

[13] L. Perko, Differential equations and dynamical systems. 2nd ed, Vol. 7 of Texts in Applied Mathematics. Springer, New York (1996).

[14] A. Schmidt, Computation of three dimensional dendrites with finite elements. J. Comput. Phys. 125 (1996) $293-312$.

[15] R.F. Sekerka, Morphological instabilities during phase transformations, in Phase transformations and material instabilities in solids, Proc. Conf., Madison/Wis. 1983. Madison 52, M. Gurtin Ed., Publ. Math. Res. Cent. Univ. Wis. (1984) $147-162$.

[16] J. Strain, Velocity effects in unstable solidification. SIAM J. Appl. Math. 50 (1990) 1-15.

[17] G. Strang and G.J. Fix, An analysis of the finite element method. Prentice-Hall Series in Automatic Computation, PrenticeHall, Inc., Englewood Cliffs, N.J (1973).

[18] A. Veeser, Error estimates for semi-discrete dendritic growth. Interfaces Free Bound. 1 (1999) 227-255.

[19] A. Visintin, Models of phase transitions, Vol. 28 of Progress in Nonlinear Differential Equations and Their Applications. Birkhäuser, Boston (1996).

[20] W.P. Ziemer, Weakly Differentiable Functions, Vol. 120 of Graduate Texts in Mathematics. Springer-Verlag, New York (1989).

To access this journal online:

www.edpsciences.org 